NBER WORKING PAPER SERIES

\title{
THE IMPACT OF ORGANIZATIONAL BOUNDARIES ON HEALTHCARE COORDINATION AND UTILIZATION
}

\author{
Leila Agha \\ Keith Marzilli Ericson \\ Xiaoxi Zhao \\ Working Paper 28179 \\ http://www.nber.org/papers/w28179 \\ NATIONAL BUREAU OF ECONOMIC RESEARCH \\ 1050 Massachusetts Avenue \\ Cambridge, MA 02138 \\ December 2020, Revised September 2021
}

The authors gratefully acknowledge research support by NIH grant P30AG012810. We thank Mohan Ramanujan, Len McCain and Elizabeth Adams for their assistance obtaining and managing the data. We thank Jason Abaluck, Amitabh Chandra, Kimberley Geissler, Thomas Koch, Jennifer Kwok, James Rebitzer, Adam Sacarny, Jonathan Skinner, Douglas Staiger, Jessica Van Parys, and Annetta Zhou for helpful comments. The views expressed herein are those of the authors and do not necessarily reflect the views of the National Bureau of Economic Research.

NBER working papers are circulated for discussion and comment purposes. They have not been peer-reviewed or been subject to the review by the NBER Board of Directors that accompanies official NBER publications.

(C) 2020 by Leila Agha, Keith Marzilli Ericson, and Xiaoxi Zhao. All rights reserved. Short sections of text, not to exceed two paragraphs, may be quoted without explicit permission provided that full credit, including ( $)$ notice, is given to the source. 
The Impact of Organizational Boundaries on Healthcare Coordination and Utilization

Leila Agha, Keith Marzilli Ericson, and Xiaoxi Zhao

NBER Working Paper No. 28179

December 2020, Revised September 2021

JEL No. D23,I11,L14

\section{ABSTRACT}

We measure organizational concentration-the distribution of a patient's healthcare across organizations - to examine how firm boundaries affect healthcare efficiency. First, when patients move to regions where outpatient visits are typically concentrated within a small set of firms, their healthcare utilization falls. Second, for patients whose PCPs exit the market, switching to a PCP with 1 standard deviation higher organizational concentration reduces utilization by $21 \%$. This finding is robust to controlling for the spread of healthcare across providers. Increases in organizational concentration predict improvements in diabetes care and are not associated with greater use of emergency department or inpatient care.

Leila Agha

Department of Economics

Dartmouth College

6106 Rockefeller Hall

Hanover, NH 03755

and NBER

leila.agha@dartmouth.edu

Keith Marzilli Ericson

Boston University Questrom School of Business

595 Commonwealth Avenue

Boston, MA 02215

and NBER

kericson@bu.edu
Xiaoxi Zhao

Department of Economics, Boston University

xiaoxiz@bu.edu 


\section{Introduction}

Transaction costs and imperfect information can make it difficult to coordinate production across firm boundaries (Coase 1937; Williamson 1985). The determinants of firm boundaries have been the subject of substantial theoretical and empirical investigation, particularly in the literature on vertical integration (Lafontaine and Slade 2007). Yet, we know less about how firm boundaries affect firm performance (Mullainathan and Scharfstein 2001), and empirical studies from different industries find mixed results. ${ }^{1}$

In healthcare, the challenges of cross-firm coordination are particularly salient; patient care is often produced with the input of many healthcare providers working in separate organizations. Geographically and over time, there is substantial variation in the organizational structures those providers operate in. An increasing fraction of US physicians is employed by large practices or hospitals (Welch et al. 2013), which may mitigate these coordination challenges. Integrated care organizations such as the Mayo Clinic, Intermountain Healthcare, and Kaiser Permanente are often held up as models of clinical efficiency and coordinated care (Enthoven 2009). Yet empirical evidence on how organizational boundaries affect healthcare delivery is limited.

In this paper, we investigate how organizational boundaries affect healthcare utilization. Existing evidence has shown that when coordination of care is more difficult, healthcare utilization tends to be higher. Higher utilization can be a sign of reduced efficiency, particularly when it is not accompanied by commensurate improvements in care quality. Coordination challenges can emerge when healthcare for an individual patient is spread across many individual providers (Agha et al. 2019; Frandsen et al. 2015), or when provider teams have fewer repeat interactions (Agha et al. forthcoming, Kim et al. 2020, Chen forthcoming). Cebul et al. (2008) argue that fragmentation across organizations may also be an important source of healthcare inefficiency. Organizational boundaries can affect coordination costs; e.g., healthcare firms often restrict information transmission to external providers by limiting transfer across electronic medical record systems. Providers may invest in firm-specific relationships and infrastructure that improve productivity (Huckman and Pisano 2006). Finally, organizational fragmentation can affect incentives for clinical process improvement and other efficiency-enhancing investments due to common agency problems and spillovers that prevent firms from reaping the full benefit of their investments (Frandsen et al. 2019).

We introduce the concept of "organizational concentration," which measures the distribution of a patient's outpatient visits across organizations. A patient's healthcare has maximal

\footnotetext{
${ }^{1}$ For example, see Seru (2014); Pierce (2012); Stroebel (2016); Forbes and Lederman (2010); Forman and Gron (2011).
} 
organizational concentration if all of their outpatient care is billed by the same organization. This construct builds on earlier work studying provider concentration (Pollack et al. 2016; Agha et al. 2019). Organizational concentration describes the realized experience of a given patient, and so is distinct from market concentration measures used in antitrust research, which instead measure provider market power for pricing. Patients who receive all their healthcare from one firm will have high organizational concentration even if there are many firms in the market. Conversely, a patient may have low organizational concentration in a highly concentrated market if they receive healthcare from many different specialty practices, even if each practice has a monopoly in that specialty.

To our knowledge, we are the first paper to measure organizational concentration systematically, so we begin with a detailed descriptive analysis. Using insurance claims data for a 20\% sample of Medicare fee-for-service enrollees from 2007-2016, we construct a measure of each patient's experienced organizational concentration. There is substantial heterogeneity across regions in organizational concentration, even conditional on the spread of patient care across providers. Studying patients who move across regions, we find that moving to a location with a higher level of organizational concentration is associated with lower healthcare utilization. While these results suggest that organizational concentration leads to lower healthcare spending, they should be interpreted with caution because other attributes of regional practice style and place effects may be correlated with the level of organizational concentration.

To isolate variation in organizational concentration from other aspects of the local practice environment, we exploit quasi-experimental variation in patient assignment to physicians generated by physician exits. We examine the experiences of patients whose primary care provider (PCP) exits the local market, either due to a move or retirement, following recent work by Fadlon and Van Parys (2020) and Kwok (2019). Since patients may endogenously sort to new PCPs on the basis of changes in their health status, we use an instrumental variable strategy that leverages mean reversion to predict the change in a patient's assigned PCP's average organizational concentration, adapting the approach used by Laird and Nielsen (2017) and Abaluck et al. (2021). When PCPs with low organizational concentration exit the market, their patients switch to more typical PCPs with higher average concentration and subsequently experience lower healthcare utilization. Using this variation, we estimate that patients who switch to a PCP with 1 SD higher organizational concentration have approximately $21 \%$ lower healthcare utilization in our preferred, most controlled specification. This finding is robust to controlling for the number and types of providers that the patient visits.

Our results indicate that organizational boundaries contribute additional frictions that 
drive increased healthcare utilization, and this pattern does not simply reflect the challenges of spreading care across multiple providers. Although we cannot fully isolate a PCP's tendency for organizational concentration from every other possible dimension of PCP practice style, our estimated effect remains large in specifications that control for the spread of patient care across providers, the size of the PCP's practice group, as well as other PCP characteristics (residency training, experience, gender). To the extent that observable variables are informative about selection on unobservables, this supports the claim that organizational concentration is an important independent contributor to spending variation (Oster 2019).

Finally, we investigate how organizational concentration influences quality of care. We use several measures related to distinct dimensions of healthcare quality, spanning gaps in primary care, appropriate management of chronic conditions, and use of testing and imaging. We find no strong evidence that changes in PCP organizational concentration predict changes in inpatient or emergency department visits. For patients with a chronic condition (diabetes), switching to a PCP with higher levels of organizational concentration leads to better adherence to recommended care guidelines. This finding from diabetes care provides suggestive evidence that greater organizational concentration may facilitate improved management of chronic conditions.

Taken together, our findings suggest that lower organizational concentration worsens care efficiency: when patient care frequently crosses firm boundaries, utilization increases with no evidence of offsetting quality improvements. This pattern may be a sign of the coordination difficulties associated with low organizational concentration.

High levels of organizational concentration arise when most of the providers a patient consults are integrated within the same firm. Our research is motivated by earlier work finding the effects of firm integration on productive efficiency are theoretically ambiguous. Bringing transactions into the same firm could improve communication (Arrow 1975) and reduce contracting barriers (Hart and Moore 1990; Hart and Holmstrom 2010). On the other hand, integration may also lead resources within the firm to be allocated less efficiently (Alonso et al. 2008; Friebel and Raith 2010). Moreover, integration may improve coordination in stable environments but lead to worse adaptation to change (Dessein 2014).

Empirical evidence from other industries on how integration affects firm performance has found mixed results. Mullainathan and Scharfstein (2001), Seru (2014), and Pierce (2012) document downsides to integration including less efficient capacity management, lower innovation, and insufficient knowledge sharing. By contrast, Stroebel (2016), Forbes and Lederman (2010), Forman and Gron (2011) find benefits of firm integration including superior information, better performance, and faster technology adoption. Atalay et al. (2014) argue that integration facilitates the efficient intrafirm transfer of intangible inputs, such as high 
quality managerial oversight and planning. We build on this literature by studying how firm boundaries affect health care delivery, a setting where the potential benefits of improved coordination, knowledge-sharing, and management are high, and rich insurance claims data allows us to track the production process.

Within healthcare, there is limited evidence on how the integration of healthcare providers affects care delivery. Although large consolidated practice groups argue they can deliver lower cost, higher quality healthcare by improving coordination, leveraging returns to specialization, and facilitating fixed cost investments, empirical evidence of these benefits is limited (Cutler and Scott Morton 2013). Recent work suggests that hospital mergers and acquisitions of physician practices do not spur improvements in clinical quality or health outcomes (Beaulieu et al. 2020; Koch et al. 2018). ${ }^{2}$ We build on this research by studying changes in the extent to which individual patient care crosses firm boundaries, rather than focusing on short-run effects of mergers and acquisitions. Care coordination depends on the ease of communication across multiple providers who treat the same patient, but mergers may simply bring competing providers - who rarely would have treated the same patient - into the same firm. Further, the process of organizational transformation is often slow. Because this paper does not focus on short-run effects of mergers, the effects we study may reflect longer-run operational changes associated with integration.

This paper is also related to a growing literature investigating differences in practice patterns across individual physicians. Across a variety of care contexts, individual physician quality and practice style have important effects on care outcomes. ${ }^{3}$ Recent work by Kwok (2019) and Fadlon and Van Parys (2020) documents that primary care physicians in particular have substantial influence on patients' healthcare spending. We build on this insight by investigating one important dimension of PCP practice environment and referral patterns, i.e. the PCP's tendency to concentrate patient care within organizations.

The paper is organized as follows. Section 1 introduces our measure of organizational concentration. Section 2 describes our data and sample selection. Section 3 reports descriptive statistics on regional variation in organizational concentration and uses movers between regions to explore how regional variation in organizational concentration may contribute to regional variation in healthcare utilization. Section 4 lays out our main empirical strategy exploiting PCP exits to explore the impact of organizational concentration. Section 5 presents the results on how healthcare utilization and quality outcomes change when a pa-

\footnotetext{
${ }^{2}$ These acquisitions may even raise healthcare spending, as physicians shift the site of care from doctors' offices to hospital outpatient settings (Koch et al. 2017) and exploit reimbursement rules that allow hospitalowned physician practices to charge additional facility fees (Capps et al. 2018; Whaley et al. 2021).

${ }^{3}$ For example, see Gowrisankaran et al. (2017); Molitor (2018); Chan et al. (2019); Currie and MacLeod (2017); Currie et al. (2016); Sahni et al. (2016).
} 
tient switches to a PCP with a different level of organizational concentration. Section 6 concludes.

\section{Defining Organizational Concentration}

In this project, we study the coordination frictions that arise when healthcare is spread across organizational boundaries. To do so, we define organizational concentration, adapting a concentration index that has been used in prior literature to measure the spread of patient care across providers. ${ }^{4}$ Specifically, we use a Herfindahl-Hirschman Index (HHI) that calculates how outpatient healthcare received by a patient is spread across organizations. We measure organizational concentration using outpatient care, following previous literature defining continuity of outpatient care across individual physicians (Nyweide and Bynum 2017; Nyweide et al. 2013). This allows us to consider the impact of outpatient organizational concentration on the likelihood that a patient requires an emergency department visit or hospitalization.

We calculate patient $i$ 's share of outpatient visits at each organization $j$, in a year $t$. Organizational concentration is then defined as the sum of squared shares across all the organizations:

$$
\operatorname{OrgConc}_{i t}=\sum_{j} \operatorname{share}_{i j t}^{2}
$$

In general, organizational concentration is higher when a patient visits fewer organizations. When a patient's outpatient visits are uniformly distributed across $N$ organizations, this measure is simply $1 / N$. When a patient receives all the visits from one organization, this concentration measure will be 1 . Lower values correspond to patient care that is spread more diffusely across organizations.

\section{Defining provider concentration}

To distinguish our findings from prior analyses, we will study variation in organizational concentration conditional on provider concentration. Provider concentration measures the spread of patient healthcare across providers. Following Agha et al. (2019), we construct a measure of provider care concentration where the share $_{i p t}$ measures the share of patient $i$ 's outpatient visits in year $t$ for each provider $p$ :

$$
\operatorname{ProvConc}_{i t}=\sum_{p} \operatorname{share}_{i p t}^{2}
$$

\footnotetext{
${ }^{4}$ Pollack et al. (2016) provides an overview and comparison of commonly used measures of care continuity.
} 
This measure will capture the challenges of coordinating healthcare across many providers, thus allowing us to distinguish them from the frictions that are specific to crossing organizational boundaries.

\section{Data and Sample Construction}

\subsection{Patient sample selection}

Our primary source of data is a $20 \%$ sample of Medicare Fee-For-Service (FFS) Part A and Part B claims data from 2007-2016. The 10-year panel data allows us to observe both patient moves and PCP exits. We use the Carrier, Inpatient, and Outpatient claims files to measure care utilization and spending. ${ }^{5}$ Patient demographics (age, sex, zip code) and chronic conditions are extracted from the Master Beneficiary Summary file with the Chronic Condition segment. In the remainder of this section, we describe the sample restrictions implemented to construct our main analytic samples.

\section{Initial sample restrictions}

We restrict our sample to Medicare beneficiaries who are 65-99 years old (inclusive) and continuously enrolled in Medicare FFS. After these restrictions, our data covers 9,675,113 beneficiaries. Our organizational concentration measure is defined based on Carrier file claims with an outpatient place of service; we drop 417,638 beneficiaries who did not have any visits of this type. This comprises our Broad Sample. From this Broad Sample, we define two separate analytic samples for different purposes. First, we define a "Patient Mover Sample" for a descriptive analysis studying regional variation in organizational concentration. Second, we define a "PCP Exit Sample" for our primary analysis studying the relationship between PCPs' organizational concentration and patient care utilization. We describe each of these samples below.

\section{Patient Mover Sample}

We construct a Patient Mover Sample for our initial descriptive analysis. Sample restrictions defined here follow the construction process outlined in Agha et al. (2019). We assigned each patient to a hospital referral region (HRR) on an annual basis, using the zip code reported

\footnotetext{
${ }^{5}$ The Inpatient file contains institutional inpatient claims, and the Outpatient file contains claims from institutional outpatient providers such as hospital outpatient departments or community mental health centers. The Carrier file contains non-institutional claims billed by individual providers such as physicians, and these claims can result from services provided at either outpatient or inpatient settings.
} 
in the Beneficiary Summary File. Further, we require that the patient received at least $75 \%$ of billed claims within that HRR; we drop beneficiaries who do not meet this requirement. To be included as a mover, the patient's HRR must have changed once (and only once) in our 10-year period. Further, the beneficiary must be continuously in the sample from two years before their move to two years after. Our sample includes all 25,814 moving patients who meet these criteria.

\section{PCP Exit Sample}

We construct a PCP Exit Sample for our main analysis. This analysis focuses on beneficiaries who change their attributed PCP due to the original PCP's relocation or retirement. We attribute each patient to their plurality PCP in each year, defined as the internist, family practice physician, or medical specialist who bills a plurality of the patient's Evaluation \& Management (E\&M) visits that year; ties are broken randomly. We exclude patients who have no E\&M visits and thus cannot be matched to a provider, as well as patients whose plurality provider is a surgeon or non-physician. If a patient cannot be matched to a PCP according to this algorithm, they will be excluded from the PCP Exit Sample. For more details on the PCP attribution, see Appendix A.1.

We limit this analysis to patients whose initial attributed PCP either moved (i.e. relocated once to a different HRR) or retired (i.e. bills no further Medicare claims). We also exclude patients who move across HRRs themselves. We require that the patient have the same plurality PCP for four years prior to the PCP's exit, limiting the sample to exits that occur in 2011-2016 to ensure four years of pre-exit observations. In addition to the four years of pre-exit observations for every patient, we also include up to four years of data following the exit. The PCP Exit Sample includes 51,570 beneficiaries. These patients are initially attributed to one of 25,650 exiting PCPs; including both the exiting PCPs and the destination PCPs, this sample covers 72,231 PCPs.

\subsection{Measuring organizational concentration}

\section{Measuring Organizations}

The next step is to construct our measure of organizational concentration. We begin by identifying provider organizations delivering outpatient care to each patient. We limit to provider services billed in the Carrier claims file and provided in an outpatient setting. ${ }^{6}$ We then define a visit by aggregating claims to a unique provider-date pair.

\footnotetext{
${ }^{6}$ For details on how we identify relevant claims, see Appendix A.1.
} 
We use the federal tax ID numbers (TINs) associated with each Carrier file claim to identify provider organizations. Our sample covers 447,009 TINs. TINs provide a measure of financial organization, with integrated physician practices typically billing under a unique TIN, although some large provider groups may organize themselves into subsidiaries, billing under separate TINs (Baker et al. 2016). In these cases, TINs may still delineate organizational boundaries within the firm, even though they are not a perfect measure of firm boundaries.

We find that our baseline TIN-based measure of organizational concentration is highly correlated with an alternative definition based on physicians' reported organizational ties in the CMS Physician Compare database. Physician Compare data is only available for the final three years of our sample (2014-2016), so we cannot use it as our baseline analysis, which tracks organizational concentration over a longer time period. In years where both measures are available, we use the affiliations reported in Physician Compare to construct an alternative measure of organizational ties, and compare this to our baseline TIN-based definition. The organizational concentration measures are correlated at 0.95 when averaged at the HRR level, and are correlated at 0.85 when averaged at the PCP level. For more detail comparing these measures of organizational ties, see Appendix A.2.

Earlier work by Baker et al. (2014), Austin and Baker (2015) and Baker et al. (2020) has also used TINs to measure local competition across physician provider groups. This research has shown that areas with higher market concentration pay higher prices for physician services. While this prior work suggests that providers sharing the same TINs are able to leverage oligopoly power in areas with high market concentration, our paper will test whether TIN-based measures of business organization are predictive of clinical integration that may yield offsetting benefits for patients and payers.

\section{Aggregating organizational concentration to the PCP-level and HRR-level}

Defining HRR-level concentration. We calculate organizational concentration at the patient-year level following the definition in equation 1 . To characterize the pattern of organizational concentration at the hospital referral region (HRR) level, we simply average the patient-level measures across all patients within the relevant region. To construct these regional averages, we use the broad sample excluding individuals who move between HRRs at any point.

Defining PCP-level concentration. Our primary empirical strategy exploits variation in PCPs' tendencies towards organizational concentration. To construct these PCP averages, we use the broad sample excluding individuals who do not match to a PCP. 
To account for statistical noise in PCP organizational concentration, we apply a conventional empirical Bayes correction (Morris 1983). This correction shrinks the estimated PCP concentration towards the doctor's mean, in proportion to the amount of estimation error. ${ }^{7}$ Since we require that patients match to their exiting PCP for four years prior to the exit, we use a four-year history to define the PCP's organizational concentration style. The average patient in our broad sample is seen by a PCP who has 144 other attributed patients over the lookback period.

To investigate the degree of shrinkage, we calculate "pseudo shrinkage coefficients" for organizational concentration, defined as each physician's demeaned Bayesian posterior divided by the demeaned raw (not shrunk) estimate. A coefficient of one implies no shrinkage. The median coefficient is 0.97 , with the 10 th percentile at 0.83 . This distribution suggests relatively little shrinkage, consistent with the high correlation (0.98) between the raw and shrunk measures.

For regression analyses at the HRR and PCP level, we exclusively rely on jackknifed versions of these organizational concentration measures that omit the index patient to avoid bias driven by an individual patient's need for more specialized care.

Paralleling the procedure for organizational concentration, we calculate HRR and PCP level measures of provider concentration to include in some regressions. This measure is also jackknifed, and the PCP level provider concentration is shrunk with an empirical Bayes procedure.

\subsection{Outcome measures}

Our primary outcome variable is a patient's annual healthcare utilization, which aggregates a patient's spending across the Medicare Inpatient, Outpatient and Carrier claim files. Utilization measures are constructed using a fixed set of annual Medicare prices expunged of regional price adjusters. ${ }^{8}$

We also study the relationship between organizational concentration and several utilizationbased measures of healthcare quality. We study two measures related to the use of hospital care: a binary indicator for any inpatient hospitalization, and a binary indicator for any

\footnotetext{
${ }^{7}$ To implement the empirical Bayes correction, we estimate a random effects model where patient-level organizational concentration depends on year fixed effects and PCP-year random effects. To achieve jackknifing, we omit the index patient from this regression. We recover empirical Bayes estimates of PCP-year organizational concentration as the sum of the year fixed effect and the best linear unbiased predictor of the PCP-year random effect.

${ }^{8}$ Medicare prices include some regional adjustments on the basis of local wage indices, and we do not want this source of regional variation in wage indices to confound the relationship between organizational concentration and spending. Following Finkelstein et al. (2016), we adjust total spending to strip away variation that is due to regional price adjustments.
} 
emergency department (ED) visit. We also examine the effects of organizational concentration on the indicators of healthcare quality for patients with diabetes: receipt of an HbA1c test and LDL test. These outcomes are only defined for the sub-sample of patients with diabetes, as defined by the Chronic Condition Warehouse; tests are identified with HCPCS codes. Finally, a potential cost of poorly coordinated care is low-value imaging and laboratory tests; we look at total utilization of these services. See Appendix A.1 for further details on data construction.

\section{Descriptive Evidence on Organizational Concentra- tion}

\subsection{Summary statistics}

Table 1 provides summary statistics for the Broad Sample (column 1), the Patient Mover Sample (column 2), and the PCP Exit Sample (column 3). The average level of organizational concentration is about 0.45 in all three samples, demonstrating that most patients regularly seek outpatient care across multiple organizations. The average level of provider concentration is slightly lower, which we would expect, given that patients will often see multiple providers within the same organization. In the Broad Sample and Patient Mover Sample, average care utilization is about $\$ 9000$ per year; utilization is lower in the PCP Exit Sample, perhaps in part due to the disruptive impact of PCP exits. Patients in the Patient Mover and PCP Exit samples are older on average than in the Broad Sample, likely because they must be observed for a few years in sample prior to the move or exit event.

We use standard deviations of the patient-level, PCP level, and HRR level measures

of organizational concentration to interpret the scale of our regression results. Appendix Table A1 further reports the mean and standard deviation of organizational concentration, provider concentration, and total utilization at these different levels. The raw standard deviation of organizational concentration across PCPs reported there (0.108) is slightly larger than the standard deviation after Empirical Bayes shrinkage (0.096); the estimates are very close, consistent with minimal shrinkage. To interpret the scale of our subsequent results, we reference the 0.1 estimated standard deviation of organizational concentration across PCPs.

We examine how organizational concentration varies across regions in Figure 1. Large variation between regions in healthcare usage suggests that some regions may be inefficient (Skinner 2011), and prior research has sought to explain why this variation exists (e.g. Cutler et al. 2019; Molitor 2018; Frakes 2013; Finkelstein et al. 2016). Our figure displays residual variation in organizational concentration across regions, after accounting for the role 
of provider concentration, age, sex, and race. As shown in the map, the West and Upper Midwest have higher organizational concentration than would be predicted by their provider concentration and demographics, while the South and Mid Atlantic have lower organizational concentration.

Figure 2 shows binned scatter plots relating organizational concentration and total healthcare utilization. Panel A shows the relationship between these variables averaged at the regional (HRR) level, while Panel $\mathrm{B}$ shows the relationship at the PCP-level. Panel A illustrates that regions with higher organizational concentration have lower levels of care utilization on average, while Panel B shows that patients of PCPs with higher organizational concentration have lower levels of healthcare utilization. The patterns shown in these graphs motivate our analytic approach, as they suggest a link between organizational concentration and care utilization. An alternative version of these graphs shown in Appendix Figure A1 demonstrates that the negative relationship between organizational concentration and utilization persists after controlling for provider concentration.

\subsection{Regional variation in organizational concentration and patient moves}

Previous work has examined patients who move between regions to identify the effect of regional practice variation on spending (Finkelstein et al. 2016; Agha et al. 2019). Here, we use the same mover design to examine how changes in regional organizational concentration are associated with healthcare utilization of patients who move. When moving between regions, patients are exposed to a change in the local pattern of organizational concentration. Following prior work, we run regressions of the form:

$$
Y_{i t}=\lambda_{O} \Delta \operatorname{OrgConc}_{i}^{H R R} \times \text { post }_{i t}+\lambda_{P} \Delta \operatorname{ProvConc}_{i}^{H R R} \times \text { post }_{i t}+X_{i t}^{\prime} \gamma+\varepsilon_{i t}
$$

where $Y_{i t}$ is the outcome of interest, $\triangle \operatorname{Org} \operatorname{Conc}_{i}^{H R R}$ is the change in regional organizational concentration experienced when patient $i$ moves, and $\triangle \operatorname{ProvConc}_{i}^{H R R}$ is the change in regional provider concentration experienced when the patient moves. We include in $X_{i t}$ an individual fixed effect, $\xi_{i} ; \gamma_{t}$, a year fixed effect; and $\tau_{(i, t)}$, a vector of event-time fixed effects indicating the year relative to the patient move.

Figure 3 presents event study graphs. Panel A shows that when patients move to a region with higher average organizational concentration, they experience an immediate and persistent increase in their individual organizational concentration. Table 2 reports corresponding regression results, summarizing how changes in regional average organizational concentration translate into individual patients' experiences when they move. If all regional 
variation were due to differences in the types of patients that lived in each region, then we would expect zero pass-through, while if movers fully adopted the average patterns of care in each region they lived, we would expect $100 \%$ pass-through. The regression in column 1 shows that about $80 \%$ of the regional difference in organizational concentration translates into patient-level changes in organizational concentration. These results are consistent with (Finkelstein et al. 2016), which found that place-based characteristics account for slightly more than half of regional variation in spending.

Figure 3 Panel B shows that when patients move to a region with higher average organizational concentration, they experience a decline in healthcare utilization. The final columns of Table 2 quantify this effect. Column 2 shows that moving to a region with 1 standard deviation (SD) greater regional organizational concentration (an increase of 0.05) is associated with a $5.6 \%$ decline in total utilization. However, we know that changes in regional organizational concentration are also correlated with changes in regional provider concentration. Column 3 adds a control for the region's provider concentration, and finds that the relationship between organizational concentration and total utilization diminishes only slightly: a 1 SD increase in regional organizational concentration is associated with a $3.9 \%$ decline in total utilization. These results suggest that the spread of patient care across distinct organizations is an important predictor of regional variation in health care utilization.

\section{Identification Strategy: PCP Exits}

\subsection{Motivation}

In the previous section, we described how regional variation in organizational concentration predicts spending outcomes. A challenge for interpreting these findings is that regional organizational concentration may also be correlated with other features of the local healthcare environment. To address this concern, we study PCP exits. When a PCP exits a local market, due to a retirement or long-distance move, that PCP's patients must find new care providers within their local market. This natural experiment allows us to study exogenous variation in PCP practice style holding constant many features of the local healthcare environment.

Organizational concentration may depend on a patient's PCP as a result of the PCP's referral decisions. PCPs may deliberately choose to refer preferentially to other providers within a multispecialty practice. In addition, PCPs themselves may be affiliated with a large organization that is tied to many local specialists, increasing the organizational concentration 
that would occur even without preferential referrals. We characterize each PCP's practice pattern with their average organizational concentration. We then test what happens to patient-level organizational concentration and healthcare utilization when a PCP exit forces the patient to switch to a new PCP with a different level of organizational concentration.

Our study of PCP exits thus analyzes how a change in the organizational concentration of a patient's assigned PCP affects the patient's outcomes. Because we observe patients who switch PCPs, we can include patient fixed effects in our regression model to control for any fixed patient attributes that influence their healthcare utilization. However, patients may endogenously sort to new PCPs on the basis of changes in their demand for care. For instance, patients who have gotten sicker may deliberately seek out multispecialty practices or well-known health systems when their original PCP exits. This type of sorting would bias our estimation of how organizational concentration affects healthcare spending within a difference-in-differences framework, since patient fixed effects would not adequately capture changes over time. As a result, we focus our analysis on an instrumental variables strategy adapted from Laird and Nielsen (2017) and Abaluck et al. (2021).

Our instrumental variables (IV) approach exploits the statistical property of mean reversion to predict the change in the organizational concentration of a patient's assigned PCP after their original PCP exits. Patients whose initial PCP was highly concentrated will, on average, experience a decrease in their PCP's organizational concentration when they switch providers. Patients whose initial PCP had low concentration will, on average, experience an increase in their PCP's organizational concentration.

Although the PCP exit strategy approach holds the regional practice environment fixed, PCP practice style is still multidimensional. A PCP's organizational concentration may be correlated with other aspects of the PCP's practice style. Prior research has documented that concentrating patient care within a narrow set of providers (provider concentration) is associated with lower levels of utilization (Agha et al. 2019; Hussey et al. 2014; Frandsen et al. 2015). To establish that the impact of organizational concentration is distinct from the well-studied phenomenon of provider concentration, our main regression specifications include both measures. We instrument for the change in provider concentration using an analogous approach to that used for organizational concentration: the provider concentration practice style of the exiting PCP.

\subsection{Estimating Equations}

In this section, we lay out a formal instrumental variable econometric framework adapted from Abaluck et al. (2021). We are ultimately interested in $\lambda_{O}$, the causal effect of the PCP's 
organizational concentration on patient utilization. Let $\operatorname{Org} \operatorname{Conc}_{i t}^{P C P}$ and ProvConc ${ }_{i t}^{P C P}$ be the organizational and provider concentration of patient $i$ 's PCP $p$ in year $t$. These jackknifed concentration measures will reflect a combination of the causal effect of the PCP's practice style on a patient's concentration and the endogenous sorting of patients to PCPs.

We first specify the causal effect $\beta_{p t}$ of each patient's PCP on their healthcare utilization $Y_{i p t}$, and then consider how this causal effect is related to organizational concentration. Let $D_{i p t}$ be a vector of dummy variables that report whether patient $i$ is attributed to PCP $p$ in year $t$. The following regression estimates the causal effect $\beta_{p t}$ of the PCPs themselves:

$$
Y_{i p t}=\sum_{p} \beta_{p t} D_{i p t}+X_{i p t}^{\prime} \gamma+\xi_{i}+\varepsilon_{i p t}
$$

where $\xi_{i}$ is an individual fixed effect, and $\varepsilon_{i p t}$ is an idiosyncratic error term. We have $X_{i p t}^{\prime}=\theta_{t}+\tau_{(i, t)}$, where $\theta_{t}$ are calendar year fixed effects, and $\tau_{(i, t)}$ are event time fixed effects. In some specifications, $X_{i p t}^{\prime}$ further controls for PCP characteristics such as gender, experience, training, and the number of doctors and number of claims billed to the PCP's tax ID number (as a proxy for organization size).

Then, we can write the relationship between the PCP's causal utilization effect and our concentration measures as follows:

$$
\beta_{p t}=\lambda_{O} O r g \operatorname{Conc}_{i t}^{P C P}+\lambda_{P} \operatorname{ProvConc}_{i t}^{P C P}+\eta_{p t}
$$

where $\lambda_{O}$ tells us how much a PCP's causal spending effect changes when PCP organizational concentration is higher, holding constant other PCP characteristics, and $\eta_{p t}$ contains residual unobserved variation in PCP spending effects that is unrelated to PCP concentration measures or other observed PCP characteristics. When we substitute 5 into 4 , we have:

$$
Y_{i p t}=\lambda_{O} O r g \operatorname{Conc}_{i t}^{P C P}+\lambda_{P} \operatorname{ProvCon}_{i t}^{P C P}+X_{i p t}^{\prime} \gamma+\xi_{i}+\eta_{p t}+\varepsilon_{i p t}
$$

Recall that $\xi_{i}$ is a patient fixed effect (which we will estimate) and $\eta_{p t}$ and $\varepsilon_{i p t}$ are unobserved error terms.

The endogeneity concern with a simple differences-in-differences approach is that $\varepsilon_{i p t}$ might be correlated with $\operatorname{OrgConc} c_{i t}^{P C P}$ (or ProvConc ${ }_{i t}^{P C P}$ ). For example, this correlation could arise if negative health shocks lead patients to switch to doctors with lower organizational concentration or provider concentration. To circumvent this endogeneity, we (1) select our sample to only include patients who are forced to switch providers because their original PCP retires or moves, and (2) instrument for the change in a patient's PCP's organizational concentration. 
To account for this source of endogeneity, we instrument for PCP organization organizational concentration in equation 6 with the level of patient's original PCP's organizational concentration interacted with a post-exit indicator. Let the instrument be $Z_{1 i t}=$ $\operatorname{OrgConc}_{i, \text { orig }}^{P C P} \times$ post $_{i t}$. We also instrument for the change in the PCP's provider concentration, $Z_{2 i t}=\operatorname{ProvConc}_{i, \text { orig }}^{P C P} \times$ post $_{i t}$. Let $Z_{i t}=\left[Z_{1 i t} Z_{2 i t}\right]$.

Our first stage equation is then:

$$
\operatorname{OrgConc}_{i t}^{P C P}=\Pi_{Z} Z_{i t}^{\prime}+\Pi_{X} X_{i p t}^{\prime}+\pi_{i}+\nu_{i p t}
$$

where the coefficients on the instrument, $\Pi_{Z}$, show how post-exit $\operatorname{Org} \operatorname{Conc}_{i t}^{P C P}$ is related to the patient's original PCP's concentration measures. We also estimate an additional parallel first stage equation for $\operatorname{Prov} \operatorname{Conc}_{i t}^{P C P}$.

\subsection{Identification assumptions}

The conditions below are necessary for the IV strategy to be valid. We explore the evidence supporting these assumptions in Section 5.1. To simplify notation, we define $\tilde{Z}_{1 i t}$ be the residualized instrument $Z_{1 i t}$ after partialling out $X_{i p t}$, patient fixed effects and $Z_{2 i t}$; correspondingly, $\tilde{Z}_{2 i t}$ is the residualized instrument after partialling out $X_{i p t}$, patient fixed effects, and $Z_{1 i t}$. Let $\tilde{Z}_{i t}=\left[\tilde{Z}_{1 i t} \tilde{Z}_{2 i t}\right]$.

1. First Stage Relevance: $\operatorname{Cov}\left(\tilde{Z}_{1 i t}, \operatorname{Org} \operatorname{Conc}_{i t}^{P C P}\right) \neq 0$ and $\operatorname{Cov}\left(\tilde{Z}_{2 i t}, \operatorname{Prov}_{\operatorname{Conc}}^{P C P}\right) \neq$ 0 . This is the assumption that the instruments predict the endogenous variables and satisfy the standard rank condition. In our case, we find patients whose PCPs exit switch to more typical PCPs; the first stage relationship is strong.

2. No Differential Shocks: $\operatorname{Cov}\left(\tilde{Z}_{1 i t}, \varepsilon_{i p t}\right)=0$ and $\operatorname{Cov}\left(\tilde{Z}_{2 i t}, \varepsilon_{i p t}\right)=0$. This condition requires that patients whose original PCP had high organizational or provider concentration do not have different shocks in healthcare demand after the exit than patients whose original PCP had low concentration. In other words, unobserved patient-health demand shocks cannot be correlated with the instrument. This is not directly testable, but event-study graphs are illuminating here, as they test whether patient-health utilization shocks in the years leading up to the exit are related to concentration levels of the exiting PCP.

3. No Differential PCP Selection $\operatorname{Cov}\left(\tilde{Z}_{1 i t}, \eta_{i p t}\right)=0$ and $\operatorname{Cov}\left(\tilde{Z}_{2 i t}, \eta_{i p t}\right)=0$. The residual spending effect $\eta_{i p t}$ of patient $i$ 's PCP $p$ cannot be correlated with the instrument. In our context, this combines a familiar restriction - selection on observables only_plus the fallback condition described in Abaluck et al. (2021). 
The fallback condition is necessary to interpret $\lambda_{O}$ as an estimate of the average relationship between PCP organizational concentration and patient care utilization in the population. This assumption requires that patients' second-choice PCPs need to have similar relationship between organizational concentration and utilization as their first-choice PCPs and the choices of patients whose PCPs did not exit. ${ }^{9}$

Selection on observables only is then necessary to interpret $\lambda_{O}$ as the causal effect of PCP organizational concentration on spending: further factors (besides organizational concentration) that influence a PCP's spending effect are uncorrelated with the instrument after residualizing observable control variables. It is sufficient that these characteristics be uncorrelated with PCP organizational concentration.

\section{Results}

Before discussing our IV results in detail, we begin by illustrating the variation that identifies our main finding. In Figure A2, we show that patients experience sizeable changes in PCP organizational and provider concentration after their initial PCP exits. The distribution of these changes is approximately symmetrically distributed, with similar numbers of patients experiencing increases and decreases in PCP concentration. $71 \%$ of patients in our sample switch to PCPs in a different organization when their original PCP exits. Even within firms, PCPs vary in their referral practices and their tendency to concentrate those referrals within their organization. On average, patients who switch to a new PCP organization experience a 0.075 absolute-value change in PCP organizational concentration, whereas patients who maintain the same PCP organization experience a 0.050 absolute-value change in PCP organizational concentration. This evidence suggests that much of the variation in PCP organizational concentration is at the PCP-level, and not solely a feature of firm structure.

Appendix Figure A3 builds intuition for our IV approach by showing a binned scatter plot where the x-axis is the patient's origin PCP organizational concentration and the $\mathrm{y}$ axis is the change in PCP organizational concentration after the exit. The graph shows the expected regression-to-the-mean pattern: patients whose origin PCP had high organizational concentration will, on average, experience a decline in organizational concentration after the first PCP exits.

\footnotetext{
${ }^{9}$ Note that with Abaluck et al. (2021)'s fallback condition embedded in the no differential PCP selection assumption, we do not require a monotonicity assumption. Abaluck et al. (2021) show that the fallback and monotonicity assumptions are non-nested, and that the fallback condition can be microfounded from standard discrete choice models. In our context, the alternative monotonicity assumption would require, for instance, that above-median organizational concentration PCPs receive a higher share of patients moving from above- versus below median-organizational concentration PCPs.
} 
Refining this analysis, Figure 4 provides a graphical look at our main IV specification. These binned scatterplots show the residualized value of the instrumental variable along the $\mathrm{x}$-axis and residualized outcomes on the y-axis, after conditioning on our baseline set of control variables (patient fixed effects, year fixed effects, and event time fixed effects). Panel A corresponds to our first stage regression, showing that larger values of the residualized IV predict declines in PCP organizational concentration. Patients whose origin PCPs have high organizational concentration will on average switch to a lower-concentration PCP after the origin PCP exits, and conversely for patients with low-concentration origin PCPs. Panel B corresponds to our reduced form regression, showing that larger values of the IV correspond to increases in patient care utilization. Patients whose original PCPs have high organizational concentration tend to experience increases in health care utilization. The patterns in Panels A and B are approximately symmetric across high and low concentration origin PCPs, suggesting our results are driven by responses to both increases and decreases in PCP concentration. Panel $\mathrm{C}$ corresponds to a fallback test of assumption 3; we discuss this result in more detail below.

\subsection{Tests of IV assumptions}

We will now describe the evidence supporting each of the IV assumptions laid out in Section 4.3.

First Stage Relevance: First stage relevance, as required by Assumption 1, is shown in the bottom panel of Table 3, which shows first stage results from our three main specifications. Columns 5 and 6 have identical first stage equations, with PCP organizational concentration as the only endogenous variable. In column 7, we add PCP provider concentration as an additional endogenous variable, paralleling the setup in the previous section. In column 8, we control for additional PCP characteristics, including PCP gender, specialty training, and medical school graduation year. The first stage F-tests reported in Table 3 show that the instruments are strong in all specifications.

No Differential Shocks: Recall that Assumption 2 requires that patients whose origin PCPs had different levels of concentration do not have different shocks to healthcare demand. This assumption is not directly testable, but we can explore its plausibility by investigating

pre-trends in healthcare utilization. Specifically, we consider event study figures that allow us to assess whether patients whose origin PCP had higher organizational concentration experience different shocks to healthcare demand over the years leading up to the PCP exit. Results are shown graphically in Figure 5. These graphs exploit the same variation underlying our instrumental variables approach. The endogenous variables of interest are the 
interaction of these relative event time fixed effects with the change in PCP organizational concentration (in the year after minus the year before the exit). The instrumental variables are the vector of interactions between relative event time fixed effects and the original PCP's organizational concentration.

Figure 5 illustrates that when a patient's PCP exits the local market, the patient's care outcomes shift towards the practice style of their new PCP. In Panel A, we show that if the new provider is predicted to have higher organizational concentration (so their patients receive care at fewer distinct organizations), the patient's experienced organizational concentration also increases. This establishes that PCP organizational concentration plays an important role in shaping patient-level organizational concentration, even when the patient remains in the same geographic location and maintaining the same insurance coverage. In Panel B, we show that if the new provider is predicted to have greater organizational concentration, the patient's total healthcare utilization declines.

In both panels of this graph, we note an absence of pre-trends prior to the move. This demonstrates that patients whose original PCPs have different levels of organizational concentration are not on differential trends of care utilization prior to the original PCP's exit. This pattern supports the validity of our IV strategy. We also see that in the year of the PCP exit and the year following, patients have the largest year-over-year changes on both experienced organizational concentration and utilization. The new PCP's influence may grow over time, as they gradually shape the set of referred providers that the patient consults. In subsequent years 2 through 5 , patients' care evolves to conform more closely to the practice style of their new PCP.

Appendix Figure A4 replicates this finding, but splits the sample according to the origin PCP's level of organizational concentration. Results are noisier in these subsamples, but generally consistent with the pooled results. Patients who are likely to experience a decrease in organizational concentration experience increases in care utilization (see Panel B); the converse result is shown in Panel A.

No Differential PCP Selection: Next, we consider assumptions related to the sorting of patients to PCPs. Assumption 3 requires that further factors besides organizational and provider concentration that influence a PCP's spending effect must be uncorrelated with the instrument. In the next section, we will discuss the robustness of our findings to adding controls for PCP characteristics and practice environment, including PCP gender, experience, residency training, and characteristics of the PCP's practice organization. Larger firms may hire higher quality staff, have greater capital investment, or different managerial quality; by controlling for the size of the PCP's practice organization, we can separate 
any general benefits of having a PCP who is employed by a large firm from the effects of organizational concentration. Our results remain consistent as we enrich the set of PCP characteristics included as control variables.

In addition, we explore the fallback test proposed by Abaluck et al. (2021). In the spirit of a conventional balance test, we can investigate the fallback assumption using a proxy for

$\eta_{p t}$. In the broad sample, we regress patient spending on PCP characteristics (PCP gender, experience, residency training, and characteristics of the PCP's practice organization), and calculate the fitted values. We then regress the fitted values (predicted PCP spending) on observational PCP organizational concentration. The residual of this regression (called the "forecast residual") provides a proxy for $\eta_{p t}$, measuring residual variation in PCP utilization that is predictable by PCP characteristics but orthogonal to organizational concentration. In the PCP exit sample, we then use the forecast residual as an outcome variable in a regression that parallels our reduced form specification, testing whether the forecast residual is correlated with the instrumental variable. This will test whether second-choice PCPs have "atypical residuals" $\eta_{p t}$, i.e. high spending effects given their organizational concentration. Figure 4 Panel $\mathrm{C}$ shows no significant relationship between the forecast residual and the instrumental variable, conditional on patient fixed effects, year fixed effects, and event time fixed effects.

\subsection{PCP organizational concentration and utilization}

This section reports our main findings, applying the instrumental variables strategy to investigate how PCP organizational concentration affects healthcare utilization. After discussing our baseline findings, we consider several alternative specifications, and then explore the relationship between organizational concentration and care quality.

Our IV regressions in Table 3 show that the effects of organizational concentration on utilization are large and robust to accounting for other dimensions of PCP practice style, training, and practice setting. We instrument for PCP organizational concentration with the original PCP's level of organizational concentration times a post-PCP-exit indicator. Corresponding reduced form regressions are reported in Appendix Table A2 Panel A. These reduced form regressions show a positive relationship between the instrumental variable and patient's care utilization: patients whose original PCPs have higher organizational fragmentation experience an increase in their care utilization after the original PCP exits.

The estimated first stage equation in Table 3 specification 5 is strong, and shows that coming from an origin PCP with a 0.1 higher (one SD) organizational concentration predicts the patient will experience a 0.035 relative decline in PCP organizational concentration after 
the exit. ${ }^{10}$ The associated second stage with this specification in Column 1 finds that about $58 \%$ of the variation in PCP organizational concentration practice style translates into the patient's individually experienced organizational concentration.

Columns 2-4 contain our main IV results relating organizational concentration to care utilization, while columns 6 - 8 contain the associated first stage equation. ${ }^{11}$ Column 2 shows that a 0.1 increase in organizational concentration leads to a $19 \%$ decline in healthcare utilization. ${ }^{12}$ Column 3 shows that this effect attenuates only slightly when provider concentration is included as an additional endogenous variable. Though the standard error on the estimate doubles, the relationship between organization concentration and care utilization remains large and statistically significant at the $1 \%$ level. This result shows that the frictions that arise when care crosses firm boundaries are distinct from previously studied concepts of provider concentration.

The main hurdle to interpreting this relationship as the causal effect of organizational concentration is that PCPs with more concentrated practice styles may differ along other dimensions besides their organizational concentration. By focusing on PCP exits experienced by patients who are not themselves moving, we are able to hold constant many features of the local healthcare environment. Nevertheless, PCPs' training, organization size, and taste for aggressive care may covary with the PCP's tendency to concentrate care within an organization. To address this concern, we introduce controls for PCP gender, residency training, and experience (based on medical school graduation year). Further, we control for the size of the PCP's practice organization, as measured by the log number of distinct providers billing to the TIN, as well as the log number of claims billed to the TIN. By controlling for the organization size, we can account for the possibility that physicians working in larger practice groups have different quality, practice style, or access to capital inputs.

Reassuringly, we find no attenuation of the relationship between the PCP's organizational concentration and patient utilization once we account for PCP characteristics and practice size. Our preferred, most controlled specification (Column 4) shows that a 0.1 increase in PCP organizational concentration is predicted to reduce health care spending by $21 \%$. The robustness of our findings to these controls provides evidence that our results are driven by differences in organizational concentration, and are not an artifact of different practice

\footnotetext{
${ }^{10}$ In order to interpret the first stage equation, recall that the equation contains an individual patient fixed effect. A coefficient of -1 would indicate that the predicted decline in PCP's organizational concentration would move one-for-one with the origin PCP's organizational concentration, as would occur if patients sorted randomly to PCPs.

${ }^{11}$ Note that columns 5 and 6 share a common first stage since they differ only in the choice of the dependent variable, so column 6 simply repeats column 5 .

${ }^{12}$ To calculate this effect size, we use the slope coefficient of -2.120 in the following expression: $1-$ $e^{-2.120 * .1}=0.191$.
} 
settings, physician training, or experience.

\subsection{Robustness and alternative specifications}

Difference in differences results. These findings can be contrasted with the differencein-differences specifications reported in Appendix Table A2 Panel B. The difference-indifferences specification continue to include patient fixed effects and timing fixed effects. The key independent variable of interest is the change in PCP concentration (in the year after minus the year before the exit) interacted with a dummy for the post-PCP-exit period. Without the instrumental variable approach, we estimate a smaller effect of PCP organizational concentration on care utilization, although effects remain negative and statistically significant in all specifications. These results are likely attenuated due to confounding. Patients who find themselves in worsening health are more likely to seek out care at large, integrated practices that include a wide array of specialists. PCPs affiliated with these practices are likely to have higher organizational concentration, but the patients who endogeneously select them may have increasing demand for health care services. This comparison highlights the motivation behind the instrumental variables approach. Specifically, a patient's choice of new PCP after their original PCP exits is likely to be endogenous to changes in the patients' demand for care. By isolating the variation in PCP organizational concentration that is predictable due to mean reversion, the IV approach avoids relying on these endogenous selection patterns to estimate the impact of organizational concentration.

Exploring the role of PCP provider concentration. Appendix Table A3 provides more detail on our results, specifically reporting our instrumental variable results on how PCPs' provider concentration practice style affects care utilization. In column 2, we estimate an alternative specification that only includes PCP provider concentration as an endogenous variable, omitting organizational concentration from the model. As expected, patients whose PCPs tend to concentrate their patients' care within a smaller set of providers also have lower spending. This finding corroborates the pattern found in the earlier literature on provider fragmentation (Agha et al. 2019; Frandsen et al. 2015; Austin and Baker 2015), and shows that the finding holds under a new identification strategy- our instrumental variables approach. However, once we add PCP organizational concentration as an additional endogenous variable in our IV framework, the estimated effect of provider concentration attenuates, as seen in columns 3 and 4 . In our most controlled specification, we cannot reject a zero effect of provider concentration. These results suggest that some of the spending previously attributed to the spread of care across providers may have actually reflected the challenges of coordination across organizations. Accounting for the role of organizational 
coordination diminishes the role of provider concentration.

Alternative controls for PCP organizational structure. One concern for interpreting our findings is that PCPs with high organizational concentration might practice in large, multispecialty firms, which may have better care or management quality for reasons unrelated to organizational concentration. In our baseline specifications, we control for log number of doctors in and log number of claims billed by the PCP's organization, as proxies for organization size. In Appendix Table A4, we alternatively control for indicator variables for 5 quintiles of the organization's number of doctors and number of claims (in columns 2 and 3 ), as well as an indicator for whether the PCP practices in a multi-specialty firm (in column 3). Our effect size remains similar in magnitude after the addition of these controls.

Accounting for patient demand for specialized care. Appendix Table A5 establishes that the relationship we uncover is also robust to alternative approaches to accounting for the distribution of patient care across different providers. In column 2, we enrich our instrumental variable specification to include a quadratic function of PCP provider concentration as an endogenous variable (in addition to the existing linear term). To accommodate the additional endogenous variable, we also expand the set of instrumental variables to include a quadratic of origin PCP provider concentration multiplied by a post-exit indicator. This specification relaxes the functional form assumption modeling the relationship between PCP provider concentration and care utilization. The estimated relationship between PCP organizational concentration and care utilization remains nearly unchanged, compared to our baseline results.

In Appendix Table A5 column 3, we test an alternative approach to accounting for the spread of patient care across providers. This specification controls for splines in the number of generalist providers the patient sees, as well as the number of specialist providers the patient sees. The estimated effect of organizational concentration remains large and statistically significant; the point estimate is actually larger than that reported in Table 3. The larger coefficient suggests these results may in fact overstate the relationship between organizational concentration and care utilization. Specifically, patients with high organizational concentration PCPs who consult many doctors may have less underlying demand for care than patients who see more doctors with a low organizational concentration PCP. This could occur, for example, if large practices with greater organizational concentration (because they cover a wider breadth of specialists) also tend to rotate patients across providers more commonly. 


\subsection{Decomposing the effects on utilization and care quality}

We have documented that switching to a PCP with higher organizational concentration increases care utilization. In this section, we consider what forms of utilization are most responsive to this change and explore how they relate to quality of care.

Utilization-based quality of care measures. While the quality of ambulatory care is multidimensional and difficult to quantify empirically, we present evidence on a variety of measures related to the provision of high-value care (recommended monitoring of patients with diabetes), use of intensive care settings (inpatient or emergency department), and utilization of testing and imaging, which may signal deficiencies in outpatient care. Results are reported in Table 4. Regressions in this table use the most controlled specification from Table 3, including PCP provider concentration as an endogenous variable and controlling for the full set of PCP characteristics and PCP organization size.

An important pathway by which organizational concentration could reduce total spending is by reducing the use of inpatient care. Recall that we define organizational concentration solely using outpatient provider interactions. As a result, there is no direct, mechanical relationship between organizational concentration and the PCP's propensity to recommend hospitalization, since care delivered in the hospital setting will not contribute to the concentration measure. We do not find statistically significant effects of changes in organizational concentration on hospital-related outcomes, though standard errors are large: a one SD increase (0.1) in organizational concentration predicts a 0.85 percentage point decline in the probability of having any inpatient visit (95\% CI: -3.6 to +1.9 percentage points). ${ }^{13}$

Heavy use of emergency departments (EDs) is sometimes interpreted as a sign of a weak primary care relationship, since patients may substitute ED care for services that could otherwise be provided more cheaply and efficiently in an office setting. Our estimates suggest that patients switching to high organizational concentration PCPs are less likely to visit the emergency department, although the effect is only statistically significant at the $10 \%$ level.

Next, we investigate process of care measures for patients with diabetes. We rely on two quality of care measures, adapted from the HEDIS guidelines: receiving a regular HbA1c test and LDL test. Switching to a physician with 0.1 higher organizational concentration leads to a 11 percentage point increase in HbA1c testing and a 11 percentage point increase in LDL tests; these relationships are statistically significant at the $1 \%$ level. Patients with diabetes are more likely to receive guideline-concordant care when their PCP has greater organizational concentration. Recall that this specification does not simply reflect the benefits of

\footnotetext{
${ }^{13}$ Appendix Table A8 shows that a one SD increase in organizational concentration leads to an estimated $\$ 587$ reduction in inpatient spending, (95\%CI: $-\$ 1360$ to $\$ 187)$.
} 
being treated in a large practice group (which might proxy for investment in clinical decision support or other electronic reminder system), because we control for the size of the PCP's practice organization. Rather, this finding suggests that keeping the patient's primary and specialty care integrated may lead to fewer gaps in care for chronically ill patients.

Finally, we turn to testing and imaging. Using claims from the Carrier and Outpatient files, we identify utilization of testing or imaging services in an outpatient setting. (For more details on construction of this measure see Appendix A.1.) Switching to a PCP with 0.1 higher organizational concentration reduces the likelihood of having any outpatient testing and imaging spending by 3 percentage points, and reduces total utilization in this category by $12 \%$ among patients with non-zero imaging or testing claims.

In Appendix Table A6, we investigate changes in repeated imaging, which we define as imaging of the same body part with the same imaging modality repeated within 30 days. This measure is limited because it captures only images billed through the Carrier claims; some repeated images might be performed in the Outpatient hospital setting, but due to the different coding conventions across these claims, we do not include them in our measure of repeated imaging. The coefficient on repeated imaging is very imprecisely estimated relative to the mean and not significantly different from zero. These findings suggest that while reduced imaging may contribute to the utilization reductions, these reductions are not primarily driven by changes to repeated imaging tests.

Preventive care services Appendix Table A7 further investigates the relationship between organizational concentration and measures of preventive care provision, using measures of preventive care adapted from Curto et al. (2019). Three of these services are recommended for certain elderly patients by the US Preventive Services Task Force (USPSTF). ${ }^{14}$ Two of the recommended services (mammograms, prostate cancer screening) have no significant relationship with PCP organizational concentration. One recommended service (colorectal cancer screening) is performed less frequently when the patient's PCP has higher organizational concentration; the implications of this finding for care quality are ambiguous, since colorectal cancer screening is not recommended at an annual frequency for all patients. We find no significant changes in pap smears or pelvic exams, two categories of care that the USPSTF does not recommend for patients over 65. We also find no relationship between PCP organizational concentration and flu vaccination or cardiovascular screenings, neither of which are covered by a USPSTF guideline.

\footnotetext{
${ }^{14}$ For more details on the construction of these outcomes and the relevant USPSTF guidelines, see Appendix A.1.
} 
Decomposing changes in care utilization. To provide a more complete picture of the sources of utilization changes, Appendix Table A8 disaggregates our findings on the basis of the location and type of care delivered. We have defined categories of care utilization that cut across the three files from which we draw Medicare claims, aggregating institutional and provider billings where appropriate. The purpose of this decomposition is to construct five disjoint categories that together sum up to our total utilization amount: (1) care delivered in an inpatient setting, (2) care delivered in a doctor's office or outpatient hospital setting, (3) outpatient laboratory tests and diagnostic imaging, (4) care delivered in an emergency department, (5) other provider-submitted bills (including home health, dialysis, rehabilitation centers, etc.). For more details on this decomposition, see Appendix A.1.

Point estimates in column 1 suggest that higher PCP organizational concentration leads to lower utilization across all five types of care, although only the effects on emergency department utilization and outpatient testing and imaging are statistically significant at the $5 \%$ level. Along the extensive margin, switching to a more concentrated PCP reduces the probability that a patient will have any outpatient testing or imaging. There is also a small effect on the propensity to have any outpatient office visits. Along the intensive margin, switching to a more concentrated PCP significantly reduces log utilization (among those with positive utilization in that category) for every type of care except inpatient hospitalizations.

Taken together, these findings suggest that the relationship between PCP organizational concentration and total utilization is driven in part by use of outpatient services, testing, imaging, and emergency department utilization. Point estimates suggest that reduced spending on inpatient care may also be an important part of the total effect, but inpatient utilization results are not precisely estimated. These patterns suggest that when outpatient providers are integrated within a firm, patients tend to receive fewer costly services, with no offsetting increase in inpatient hospitalizations.

\section{Conclusion}

Coordination challenges arise when clinical care is split across firm boundaries. Firms may both facilitate informal relationships among care providers, as well as make firm-specific investments in coordination technology. In the healthcare setting, coordination technology could include messaging systems, investments in health information technology, and established norms for passing off patient information across providers.

Studying patients who move regions, we document that regions with higher levels of organizational concentration also have lower levels of care utilization. This pattern suggests a role for organizational concentration in explaining regional variation in healthcare spending. 
Our main analysis studies patients who stay in the same area after their PCP exits the local market due to a retirement or move. Patients who switch to a PCP with higher organizational concentration experience reductions in care utilization, relative to patients who switch to a PCP with lower organizational concentration. These relationships persist after conditioning on detailed measures of how many generalist and specialist providers the patient sees, and how concentrated the patient's care is across those providers. This evidence indicates that the organizational ties between a patient's healthcare providers have an impact on their total healthcare utilization.

Our estimated effect (approximately a 20\% decrease in utilization from a 1 SD increase in PCP organizational concentration) is large relative to other healthcare interventions. By way of comparison, Agha et al. (2019) find that moving to a region with 1 SD higher provider fragmentation increases care utilization by $10 \%$. Clemens and Gottlieb (2014) estimate that a 2 percent increase in payment rates leads to a 3 percent increase in healthcare utilization. The introduction of a major policy initiative, Accountable Care Organizations and the Medicare Shared Savings Program, led to comparatively small reductions (less than 5\%) in spending (McWilliams et al. 2018).

Although switching to a PCP with greater organizational concentration is associated with lower total utilization of physician services, we see no evidence that higher organizational concentration reduces quality of care. In fact, PCPs with greater organizational concentration perform better on these measures of effective care for patients with diabetes.

Taken together, these findings point to a potential mechanism by which higher organizational concentration lowers utilization. When providers share an organizational affiliation, they are likely to have lower barriers to information sharing and greater trust. These benefits may reduce gaps in care - e.g. resulting in better monitoring of diabetes patients - and improve hand-offs between providers. In turn, these improvements may allow providers to avoid unnecessary referrals, ensure that referred patients have already completed the requisite workup, and centralize follow-up care with the patient's PCP. Each of these effects may reduce low-value visits that generate repeated contact with specialists.

It is also worth considering alternative explanations of these findings. Large organizations may hire higher-quality physicians. Alternatively, it may be more difficult to get a timely appointment in a large, multi-specialty practice. If this were the case, we would expect that our result would attenuate when we account for the size of the PCP's organizational affiliation or an indicator for whether the PCP is part of a multispecialty practice, but our empirical estimates show no such attenuation. Even though PCPs with higher organizational concentration have lower use of outpatient services, testing, and imaging, we see no evidence of offsetting increases in inpatient or emergency department care. 
While our results suggest potential savings associated with care delivered at integrated multispecialty practices, any gains from reduced utilization would need to be weighed against the higher prices likely paid by private insurance providers to larger practices that have more bargaining power. The Medicare claims we study are paid at administratively set prices, so an investigation of countervailing price effects is beyond the scope of this paper. Another consideration is that private insurance plans may use alternative approaches to care management (e.g. high deductibles or preauthorization requirements) that might limit the utilization of low organizational concentration PCPs, attenuating the relationship described here.

Our findings illuminate the role that firm boundaries play in organizing economic activity. These results raise the question of whether mergers that create multispecialty physician practices generate savings from reduced utilization. If these gains occur, they may take time to develop as providers adapt to changing communication systems and adopt new referral patterns. Future research examining the detailed mechanisms of how these boundaries affect teamwork and care coordination may be able to show how some of the benefits of organizational concentration could be replicated without financial integration- for exam-

ple, through better integration of health information technology systems, or by co-locating distinct provider groups.

\section{References}

Abaluck, J., M. M. C. Bravo, P. Hull, and A. Starc (2021). Mortality effects and choice across private health insurance plans. Quarterly Journal of Economics 136(3), 1557-1610.

Agha, L., K. M. Ericson, K. H. Geissler, and J. B. Rebitzer (Forthcoming). Team relationships and performance: Evidence from healthcare referral networks. Management Science.

Agha, L., B. Frandsen, and J. B. Rebitzer (2019). Fragmented division of labor and healthcare costs: Evidence from moves across regions. Journal of Public Economics 169, 144-159.

Alonso, R., W. Dessein, and N. Matouschek (2008, March). When does coordination require centralization? American Economic Review 98(1), 145-179.

Arrow, K. J. (1975). Vertical integration and communication. The Bell Journal of Economics 6(1), 173-183.

Atalay, E., A. Hortaçsu, and C. Syverson (2014). Vertical integration and input flows. American Economic Review 104(4), 1120-48. 
Austin, D. R. and L. C. Baker (2015). Less physician practice competition is associated with higher prices paid for common procedures. Health Affairs 34(10), 1753-1760.

Baker, L. C., M. K. Bundorf, and D. P. Kessler (2020). Does multispecialty practice enhance physician market power? American Journal of Health Economics 6(3), 324-347.

Baker, L. C., M. K. Bundorf, and A. Royalty (2016, October). Measuring physician practice competition using Medicare data. In A. Aizcorbe, C. Baker, E. R. Berndt, and D. M. Cutler (Eds.), Measuring and Modeling Health Care Costs, pp. 351-377. University of Chicago Press.

Baker, L. C., M. K. Bundorf, A. B. Royalty, and Z. Levin (2014). Physician practice competition and prices paid by private insurers for office visits. JAMA 312(16), 1653-1662.

Beaulieu, N. D., L. S. Dafny, B. E. Landon, J. B. Dalton, I. Kuye, and J. M. McWilliams (2020). Changes in quality of care after hospital mergers and acquisitions. New England Journal of Medicine 382(1), 51-59.

Capps, C., D. Dranove, and C. Ody (2018). The effect of hospital acquisitions of physician practices on prices and spending. Journal of Health Economics 59, 139-152.

Cebul, R. D., J. B. Rebitzer, L. J. Taylor, and M. E. Votruba (2008). Organizational fragmentation and care quality in the US healthcare system. Journal of Economic Perspectives 22(4), 93-113.

Chan, David C, J., M. Gentzkow, and C. Yu (2019, November). Selection with variation in diagnostic skill: Evidence from radiologists. Working Paper 26467, National Bureau of Economic Research.

Chen, Y. (Forthcoming). Team-specific human capital and team performance: Evidence from doctors. American Economic Review.

Clemens, J. and J. D. Gottlieb (2014). Do physicians' financial incentives affect medical treatment and patient health? American Economic Review 104(4), 1320-49.

Coase, R. (1937). The theory of the firm. Economica 4(16), 386-405.

Currie, J. and W. B. MacLeod (2017). Diagnosing expertise: Human capital, decision making, and performance among physicians. Journal of labor economics 35(1), 1-43.

Currie, J., W. B. MacLeod, and J. Van Parys (2016). Provider practice style and patient health outcomes: the case of heart attacks. Journal of Health Economics 47, 64-80. 
Curto, V., L. Einav, A. Finkelstein, J. Levin, and J. Bhattacharya (2019). Health care spending and utilization in public and private medicare. American Economic Journal: Applied Economics 11(2), 302-32.

Cutler, D., J. S. Skinner, A. D. Stern, and D. Wennberg (2019). Physician beliefs and patient preferences: A new look at regional variation in health care spending. American Economic Journal: Economic Policy 11(1), 192-221.

Cutler, D. M. and F. Scott Morton (2013). Hospitals, market share, and consolidation. JAMA 310(18), 1964-1970.

Dessein, W. (2014). Incomplete contracts and firm boundaries: New directions. Journal of Law, Economics, \& Organization 30, i13-i36.

Enthoven, A. C. (2009). Integrated delivery systems: The cure for fragmentation. American Journal of Managed Care 15(12), S284.

Fadlon, I. and J. Van Parys (2020). Primary care physician practice styles and patient care: Evidence from physician exits in medicare. Journal of Health Economics 71, 102304.

Finkelstein, A., M. Gentzkow, and H. Williams (2016). Sources of Geographic Variation in Health Care: Evidence From Patient Migration. The Quarterly Journal of Economics 131(4), 1681-1726.

Forbes, S. J. and M. Lederman (2010). Does vertical integration affect firm performance? Evidence from the airline industry. The RAND Journal of Economics 41(4), 765-790. DOI: 10.1111/j.1756-2171.2010.00120.x.

Forman, C. and A. Gron (2011). Vertical integration and information technology investment in the insurance industry. Journal of Law, Economics, \& Organization 27(1), 180-218.

Frakes, M. (2013). The impact of medical liability standards on regional variations in physician behavior: Evidence from the adoption of national-standard rules. American Economic Review 103(1), 257-76.

Frandsen, B., M. Powell, and J. B. Rebitzer (2019). Sticking points: common-agency problems and contracting in the US healthcare system. The RAND Journal of Economics 50(2), 251-285.

Frandsen, B. R., K. E. Joynt, J. B. Rebitzer, and A. K. Jha (2015). Care fragmentation, quality, and costs among chronically ill patients. Am J Manag Care 21(5), 355-362. 
Friebel, G. and M. Raith (2010, May). Resource allocation and organizational form. American Economic Journal: Microeconomics 2(2), 1-33.

Geissler, K. H., B. Lubin, and K. M. M. Ericson (2020). The role of organizational affiliations in physician patient-sharing relationships. Medical Care Research and Review r7(2), 165175.

Gowrisankaran, G., K. A. Joiner, and P.-T. Léger (2017, December). Physician practice style and healthcare costs: Evidence from emergency departments. Working Paper 24155, National Bureau of Economic Research.

Hart, O. and B. Holmstrom (2010, May). A theory of firm scope. The Quarterly Journal of Economics 125(2), 483-513.

Hart, O. and J. Moore (1990, December). Property rights and the nature of the firm. Journal of Political Economy 98(6), 1119-1158.

Huckman, R. S. and G. P. Pisano (2006). The firm specificity of individual performance: Evidence from cardiac surgery. Management Science 52(4), 473-488.

Hussey, P. S., E. C. Schneider, R. S. Rudin, D. S. Fox, J. Lai, and C. E. Pollack (2014). Continuity and the costs of care for chronic disease. JAMA internal medicine 174(5), $742-748$.

Kim, S.-H., H. Song, and M. Valentine (2020). Staffing temporary teams: Understanding the effects of team familiarity and partner variety. Working paper.

Koch, T. G., B. W. Wendling, and N. E. Wilson (2017). How vertical integration affects the quantity and cost of care for medicare beneficiaries. Journal of Health Economics 52, $19-32$.

Koch, T. G., B. W. Wendling, and N. E. Wilson (2018). The effects of physician and hospital integration on medicare beneficiaries' health outcomes. Review of Economics and Statistics, 1-38.

Kwok, J. H. (2019). How do primary care physicians influence healthcare? Evidence on practice styles and switching costs from Medicare. Working Paper. https://www . jenniferkwok.com/s/JenniferKwok_PrimaryCarePhysicians_JMP.pdf.

Lafontaine, F. and M. Slade (2007, September). Vertical integration and firm boundaries: The evidence. Journal of Economic Literature 45(3), 629-685. 
Laird, J. and T. Nielsen (2017). Physician prescribing behaviors on prescription drug use and labor supply: Evidence from movers in Denmark. Working paper.

McWilliams, J. M., L. A. Hatfield, B. E. Landon, P. Hamed, and M. E. Chernew (2018). Medicare spending after 3 years of the medicare shared savings program. New England Journal of Medicine 379(12), 1139-1149. PMID: 30183495.

Molitor, D. (2018). The evolution of physician practice styles: evidence from cardiologist migration. American Economic Journal: Economic Policy 10(1), 326-56.

Morris, C. N. (1983). Parametric empirical Bayes inference: theory and applications. Journal of the American Statistical Association 78(381), 47-55.

Mullainathan, S. and D. Scharfstein (2001, May). Do firm boundaries matter? American Economic Review 91 (2), 195-199.

Nyweide, D. J., D. L. Anthony, J. P. Bynum, R. L. Strawderman, W. B. Weeks, L. P. Casalino, and E. S. Fisher (2013). Continuity of care and the risk of preventable hospitalization in older adults. JAMA Internal Medicine 173(20), 1879-1885.

Nyweide, D. J. and J. P. Bynum (2017). Relationship between continuity of ambulatory care and risk of emergency department episodes among older adults. Annals of Emergency Medicine 69(4), 407-415.

Oster, E. (2019). Unobservable selection and coefficient stability: Theory and evidence. Journal of Business 86 Economic Statistics 37(2), 187-204.

Pierce, L. (2012, February). Organizational Structure and the Limits of Knowledge Sharing: Incentive Conflict and Agency in Car Leasing. Management Science 58(6), 1106-1121.

Pollack, C. E., P. S. Hussey, R. S. Rudin, D. S. Fox, J. Lai, and E. C. Schneider (2016). Measuring care continuity: A comparison of claims-based methods. Medical care 54(5), e30.

Sahni, N. R., M. Dalton, D. M. Cutler, J. D. Birkmeyer, and A. Chandra (2016). Surgeon specialization and operative mortality in united states: Retrospective analysis. BMJ 354 , i3571.

Seru, A. (2014, February). Firm boundaries matter: Evidence from conglomerates and R\&D activity. Journal of Financial Economics 111(2), 381-405. 
Skinner, J. (2011, January). Causes and Consequences of Regional Variations in Health Care. In M. V. Pauly, T. G. Mcguire, and P. P. Barros (Eds.), Handbook of Health Economics, Volume 2 of Handbook of Health Economics, pp. 45-93. Elsevier.

Stroebel, J. (2016). Asymmetric information about collateral values. The Journal of Finance 71 (3), 1071-1112. DOI:10.1111/jofi.12288.

Welch, W. P., A. E. Cuellar, S. C. Stearns, and A. B. Bindman (2013). Proportion of physicians in large group practices continued to grow in 2009-11. Health Affairs 32(9), 1659-1666.

Whaley, C. M., X. Zhao, M. Richards, and C. L. Damberg (2021). Higher medicare spending on imaging and lab services after primary care physician group vertical integration: Study examines higher medicare spending on imaging and lab services after primary care physician group vertical integration. Health Affairs 40(5), 702-709.

Williamson, O. (1985). The Economic Institutions of Capitalism: Firms, Markets, Relational Contracting. Free Press. 
Figure 1: Variation in organizational concentration by region

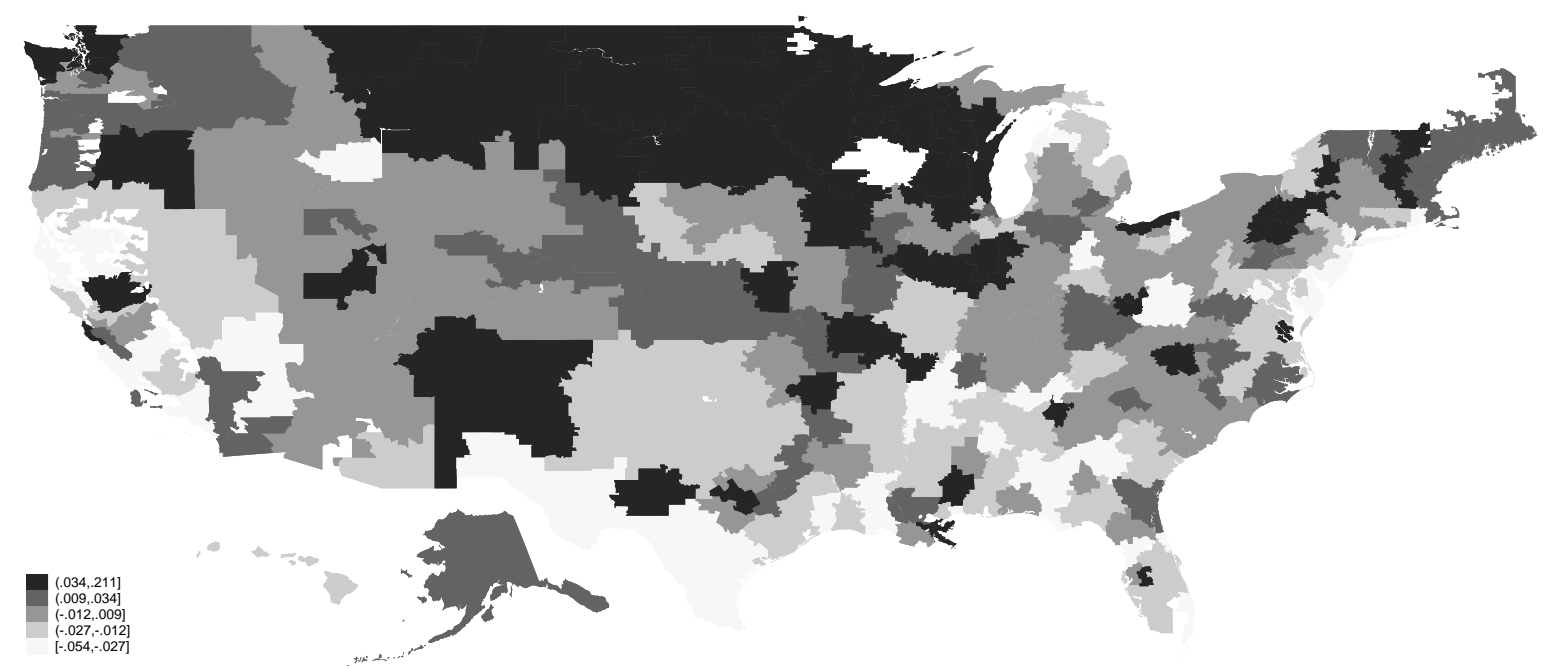

Notes: This map shows the mean residuals of patients' organizational concentration after regression adjustment for differences in average provider concentration and patient demographics (age, sex, and race). Hospital Referral Regions (HRRs) in darker gray have higher residual organizational concentration (see legend). Data: Broad Sample. 
Figure 2: Relationship between organizational concentration and healthcare utilization

(A) HRR level

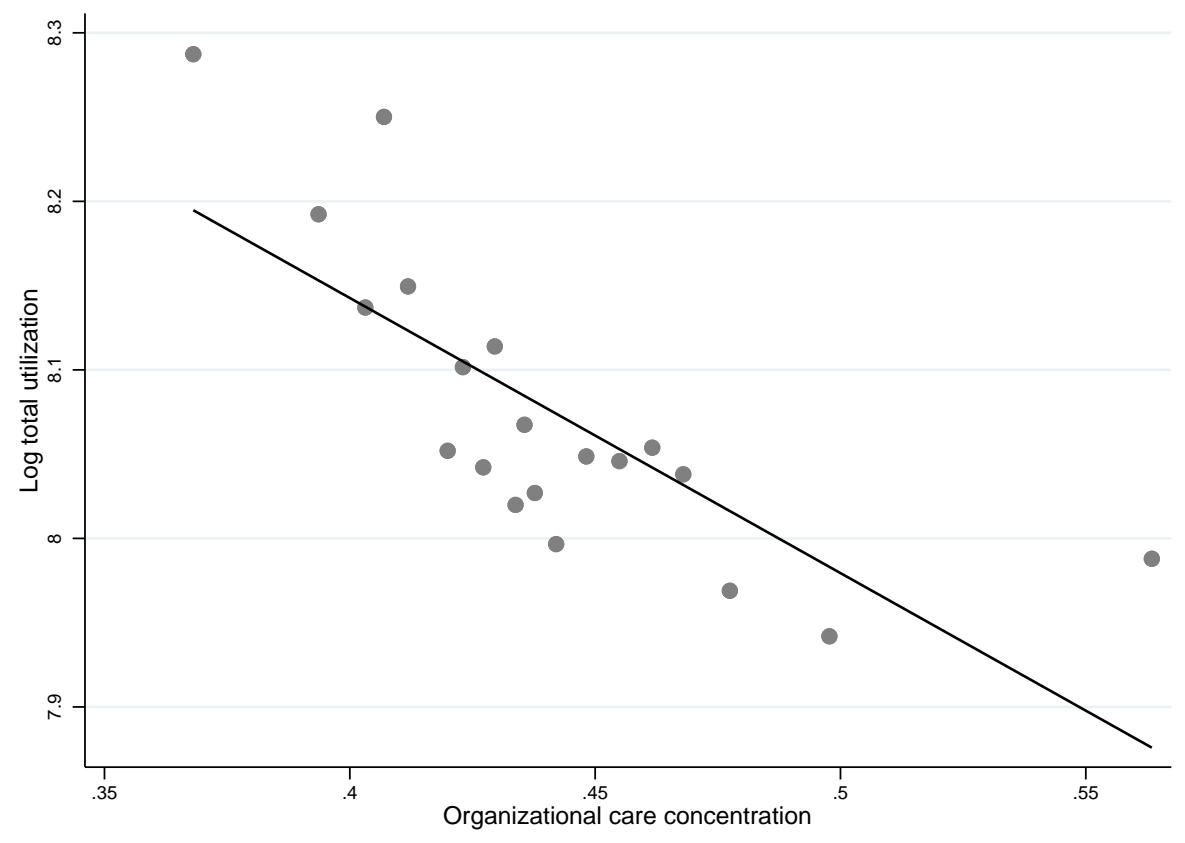

(B) PCP level

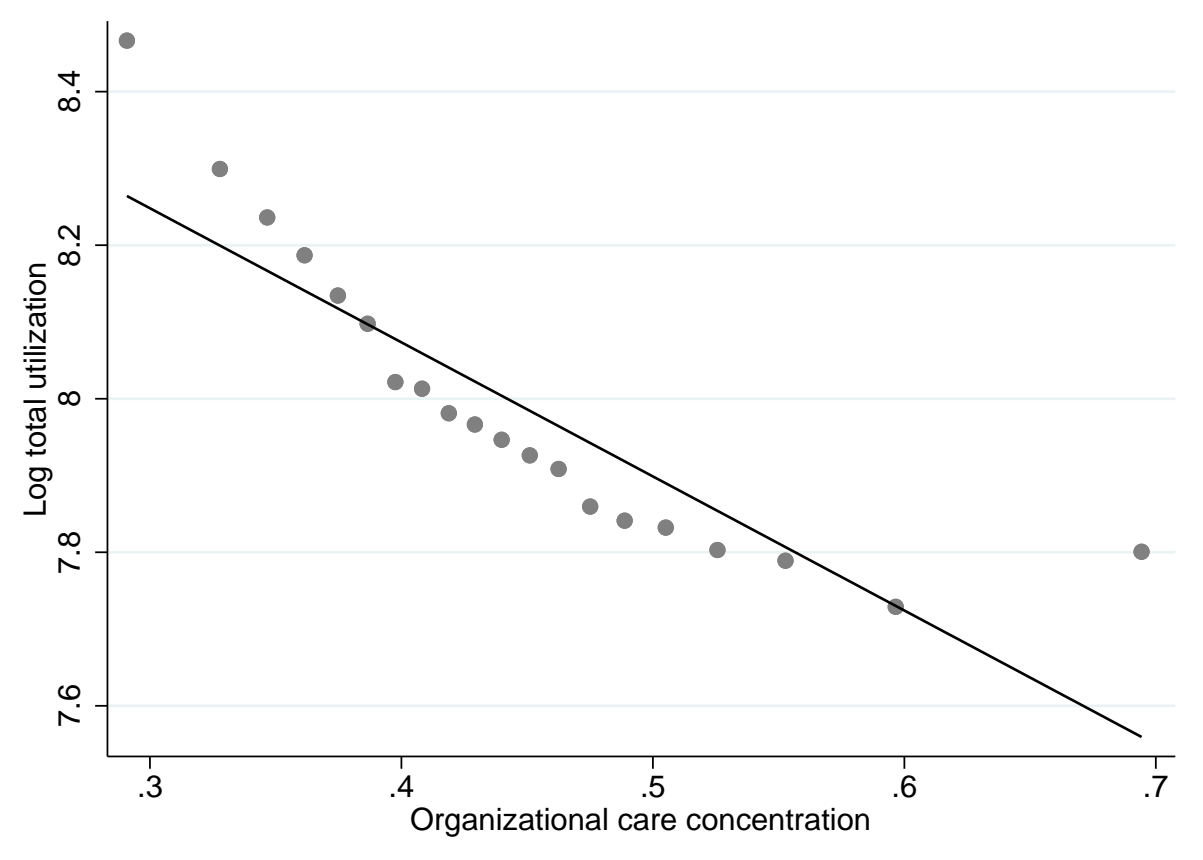

Notes: Binned scatterplots. Panel (A) shows the relationship between HRR organizational concentration and care utilization across regions. Patients are attributed to HRRs by their reported zipcode. Panel (B) shows the relationship between PCP organizational care concentration and patient utilization in the PCP Exit Sample. Patients are attributed to PCPs using the plurality assignment rule described in Section 2. 
Figure 3: Event study figures for patients who move between regions

(A) Response of patients' organizational concentration to changes in regional organizational concentration

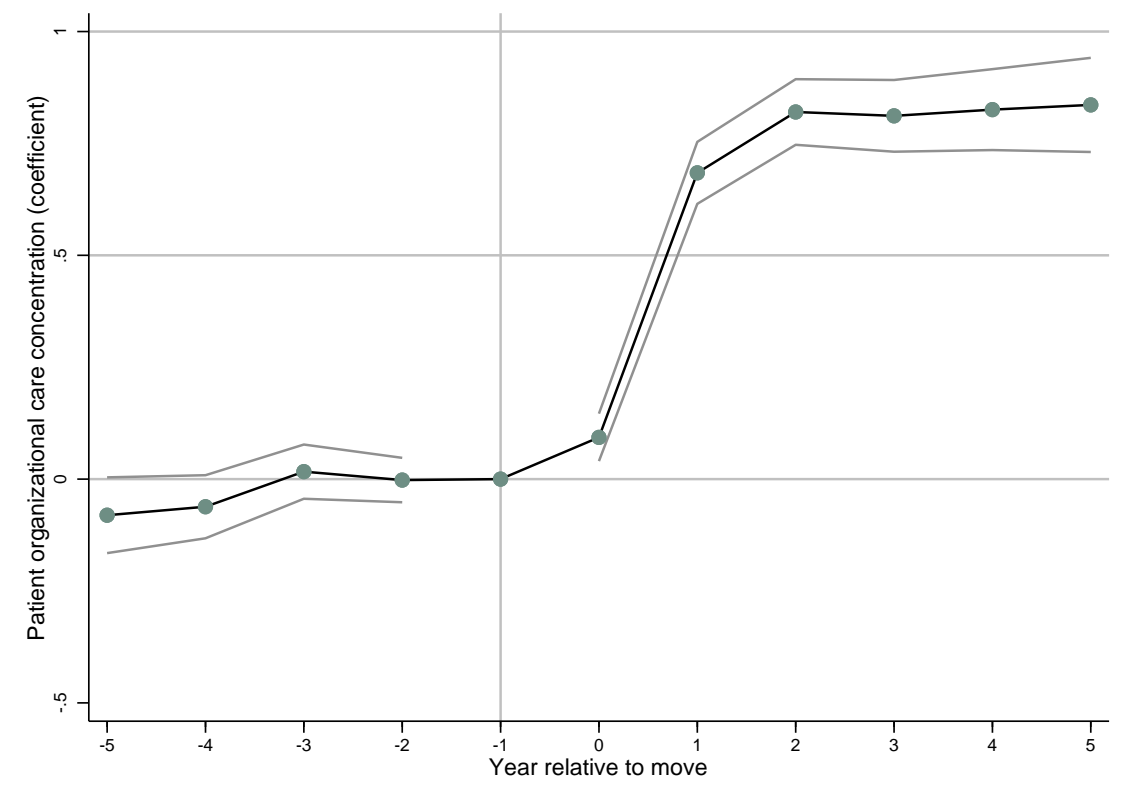

(B) Response of patients' total utilization to changes in regional organizational concentration

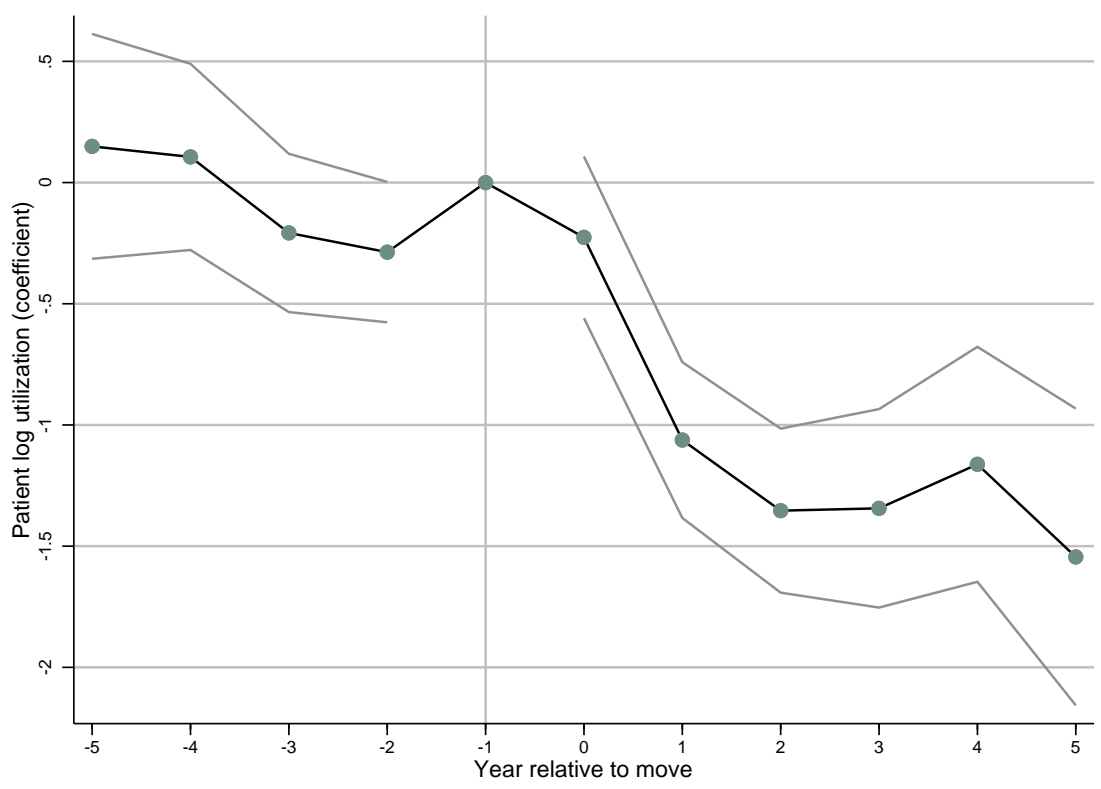

Notes: The two subplots show the estimates and 95\% confidence intervals from two separate regressions. The dependent variable in Panel A is patients' organizational concentration, and in Panel B is log utilization. The figure plots the coefficients on the change in regional organizational concentration interacted with year relative to move. Both regressions control for calendar year fixed effects, and patient fixed effects. Standard errors are clustered at HRR and patient levels. 


\section{Figure 4: Binned scatterplots illustrating the PCP exit IV strategy}

(A) First stage

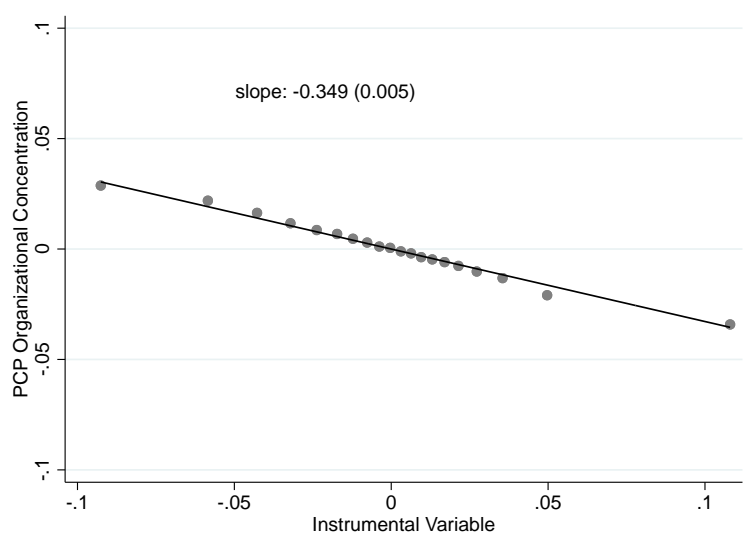

(B) Reduced Form

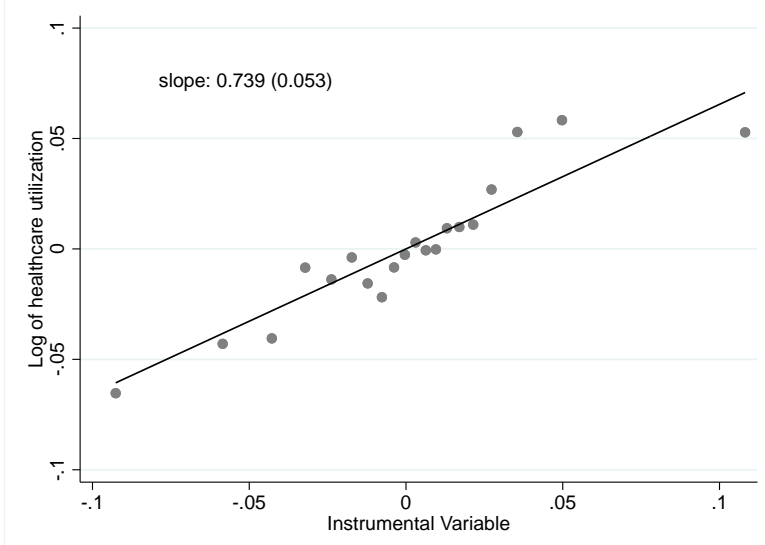

(C) Fallback

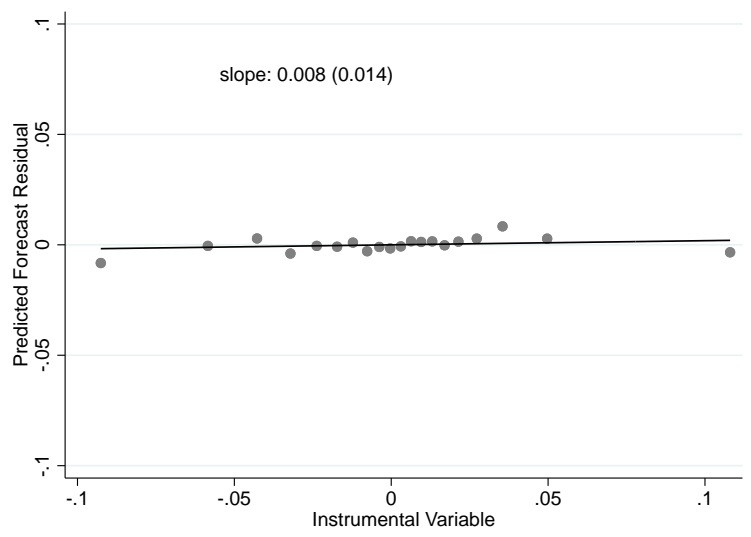

Notes: All panels residualize both dependent and independent variables to control for calendar year fixed effects, event time fixed effects, and patient fixed effects. Panel A illustrates the first stage: patients whose original PCP has higher organizational concentration on average experience a decrease in their PCP's organizational concentration after the original PCP exits. Panel B illustrates the reduced form: patients whose original PCP had higher organizational concentration on average experience an increase in total utilization. Panel C illustrates the fallback condition: original PCP concentration is uncorrelated with the forecast residual. Sample size: 304,954. 
Figure 5: Event study figures for patients whose PCP exits

(A) Response of patients' organizational concentration to changes in PCP organizational concentration

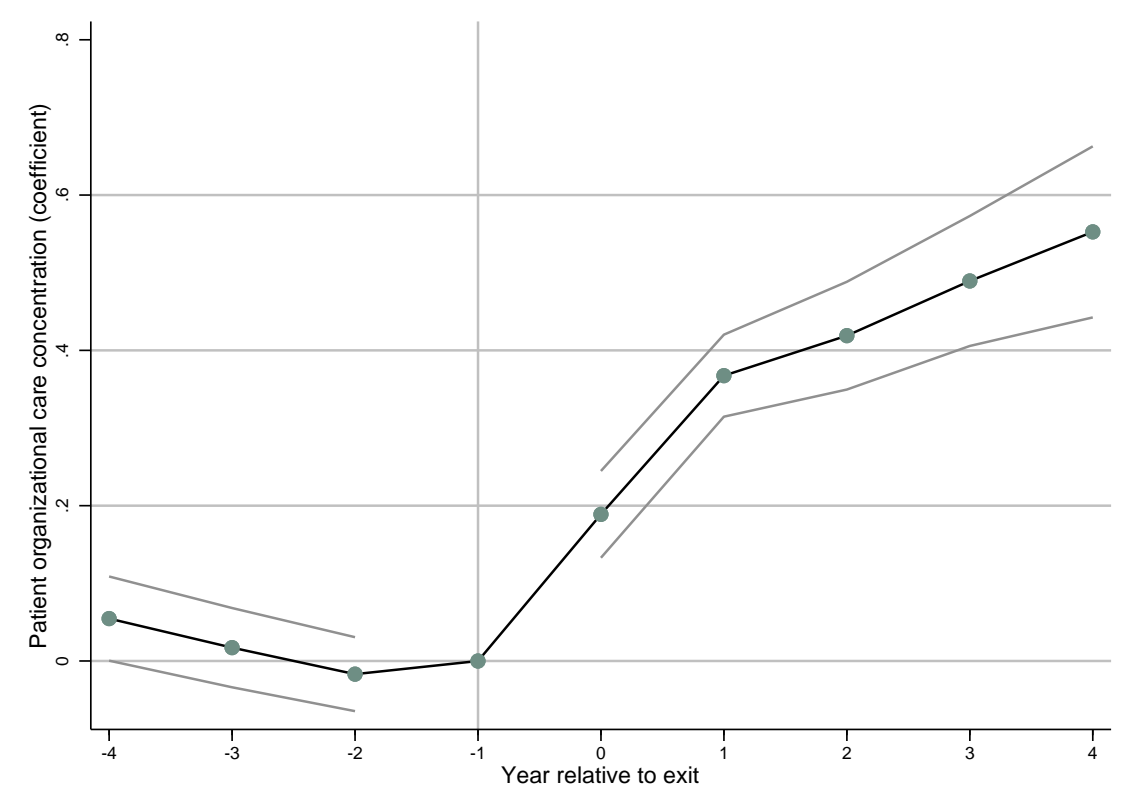

(B) Response of patients' total utilization to changes in PCP organizational concentration

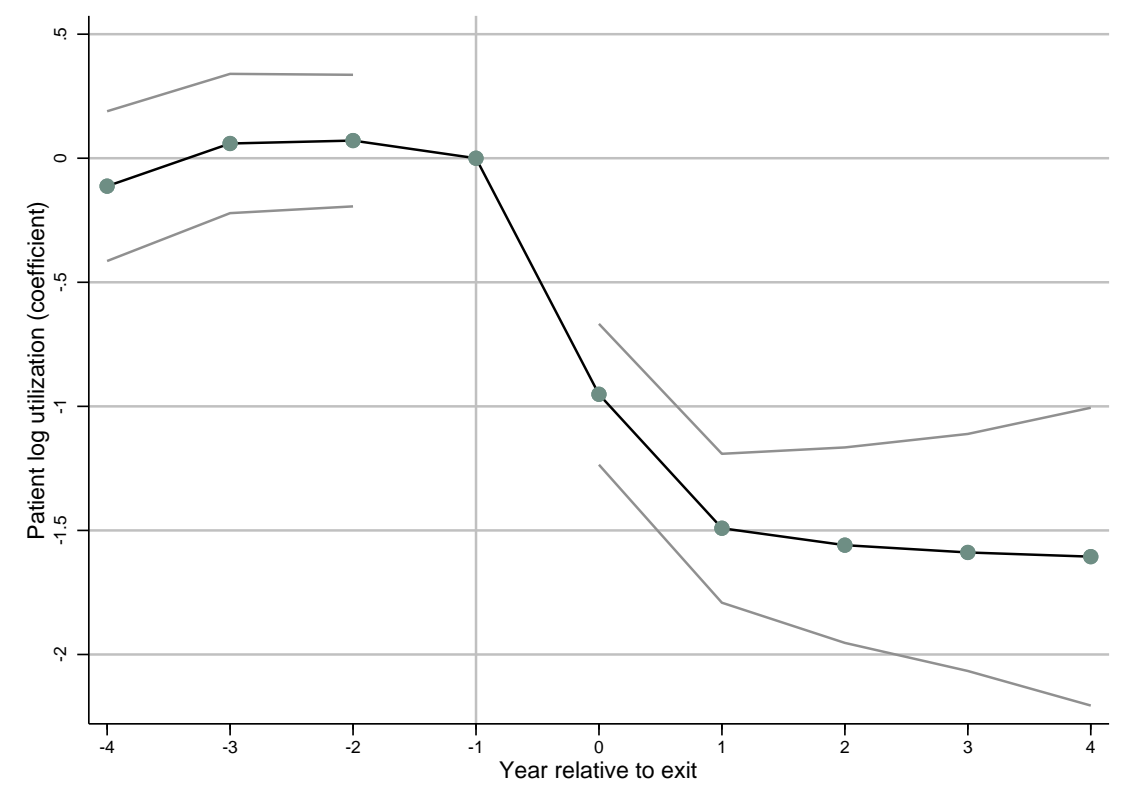

Notes: The two panels show the estimates and 95\% confidence intervals from two separate regressions. The dependent variable in Panel A is patients' organizational concentration, and in Panel B is log utilization. The endogenous variables are the interaction of these relative event time fixed effects with the change in PCP organizational concentration (in the year after minus the year before the exit). The instrumental variables are the vector of interactions between relative event time fixed effects and the origin PCP's organizational concentration. Both regressions control for calendar year fixed effects, event time fixed effects, and patient fixed effects. Because the partially treated event year 0 (year of PCP exit) is included in these specifications but excluded elsewhere, these regressions have 350,783-pbservations, slightly more than in Table 3. Standard errors are clustered at PCP and patient levels. 
Table 1: Summary statistics of different samples

\begin{tabular}{|c|c|c|c|}
\hline & $(1)$ & $(2)$ & $(3)$ \\
\hline & Broad Sample & Patient Mover Sample & PCP Exit Sample \\
\hline \multirow[t]{2}{*}{ Organizational concentration } & 0.45 & 0.44 & 0.46 \\
\hline & $(0.27)$ & $(0.26)$ & $(0.25)$ \\
\hline \multirow[t]{2}{*}{ Provider concentration } & 0.38 & 0.36 & 0.38 \\
\hline & $(0.27)$ & $(0.25)$ & $(0.24)$ \\
\hline \multirow[t]{2}{*}{ Total utilization $(\$)$} & 8696 & 8972 & 7517 \\
\hline & $(17,649)$ & $(16,239)$ & $(14,144)$ \\
\hline \multirow[t]{2}{*}{ Age } & 75.94 & 78.48 & 78.17 \\
\hline & $(7.53)$ & $(7.46)$ & $(6.96)$ \\
\hline Sex: Female & 0.58 & 0.66 & 0.62 \\
\hline Race: White & 0.87 & 0.90 & 0.87 \\
\hline Has Diabetes & 0.28 & 0.27 & 0.36 \\
\hline Has Hypertension & 0.61 & 0.65 & 0.76 \\
\hline Has Heart disease & 0.32 & 0.34 & 0.33 \\
\hline N patient-year obs & $48,604,598$ & 214,064 & 304,954 \\
\hline $\mathrm{N}$ patients & $9,177,819$ & 25,814 & 51,570 \\
\hline $\mathrm{N}$ assigned $\mathrm{PCPs}$ & & & 72,231 \\
\hline
\end{tabular}

Notes: This table reports means and then standard deviations in parentheses. Column 1 describes the Broad Sample. Column 2 describes the sample underlying our mover analysis; this sample restricts to patients who move across regions. Column 3 describes the sample underlying our analysis of PCP exits; this sample restricts to patients whose PCP exits the local market. The number of assigned PCPs in column 3 includes exiting PCPs as well as the PCPs patients switched to. 
Table 2: Patient movers and regional organizational concentration

\begin{tabular}{lccc}
\hline \hline & $(1)$ & $(2)$ & $(3)$ \\
& OrgConc $_{i t}$ & Log $\left.^{(\text {total utilization }}\right)_{i t}$ \\
\hline$\Delta$ OrgConc $_{i}^{H R R} \times$ post $_{i t}$ & $0.767^{* * *}$ & $-1.113^{* * *}$ & $-0.781^{* * *}$ \\
& $(0.028)$ & $(0.123)$ & $(0.142)$ \\
Regional provider concentration & & & $\mathrm{X}$ \\
\hline \hline
\end{tabular}

Notes: All regressions control for calendar year fixed effects, relative year fixed effects, and patient fixed effects. Regional organizational concentration and provider concentration are jackknifed. Standard errors in parentheses are clustered at the HRR and patient levels. Sample: Movers Analysis Sample, $\mathrm{N}=214,064$ patient-year observations.

*** $p<0.01,{ }^{* *} p<0.05,{ }^{*} p<0.1$ 
Table 3: The effect of organizational concentration on utilization, identified from PCP exits

\begin{tabular}{|c|c|c|c|c|}
\hline \multirow[t]{2}{*}{ Second stage } & $(1)$ & $(2)$ & $(3)$ & (4) \\
\hline & OrgConc $i t$ & \multicolumn{3}{|c|}{ Log(total utilization $)_{i t}$} \\
\hline $\operatorname{OrgConc}_{i t}^{P C P}$ & $\begin{array}{c}0.583^{* * *} \\
(0.027)\end{array}$ & $\begin{array}{c}-2.120^{* * *} \\
(0.152)\end{array}$ & $\begin{array}{c}-1.625^{* * *} \\
(0.287)\end{array}$ & $\begin{array}{c}-2.385^{* * *} \\
(0.440)\end{array}$ \\
\hline First stage & \multicolumn{4}{|c|}{$\operatorname{OrgConc}_{i t}^{P C P}$} \\
\hline $\operatorname{OrgConc}_{i, \text { orig }}^{P C P} \times$ post $_{i t}$ & $\begin{array}{c}-0.349^{* * *} \\
(0.005)\end{array}$ & $\begin{array}{c}-0.349^{* * *} \\
(0.005)\end{array}$ & $\begin{array}{c}-0.249^{* * *} \\
(0.007)\end{array}$ & $\begin{array}{c}-0.203^{* * *} \\
(0.007)\end{array}$ \\
\hline F-statistic & 33,199 & 33,199 & 8951 & 4648 \\
\hline PCP provider concentration & & & $\mathrm{X}$ & $\mathrm{X}$ \\
\hline PCP characteristics, org. size & & & & $\mathrm{X}$ \\
\hline
\end{tabular}

Notes: Each column represents an instrumental variables regression. The instrument is the exiting PCP's organizational concentration (jackknifed) multiplied by a post-exit indicator. Specification 1 's outcome variable is the individual patient's realized organizational concentration. Specifications 2-4's outcome variable is the patient's log total utilization. All regressions control for calendar year fixed effects, relative year fixed effects, and patient fixed effects. Specifications (3) and (4) include PCP provider concentration as an additional endogenous variable, instrumented by the original PCP's provider concentration multiplied by a post indicator. Specification (4) controls for PCP characteristics: gender, experience quartile indicators, residency training indicators (internal medicine vs. family practice), and the PCP's organization size (log total number of claims billed to the PCP's TIN, and the log number of unique providers billing to the PCP's TIN). Standard errors in parentheses are clustered at the PCP and patient levels. Cragg-Donald Wald F-test reported for first-stage. The PCP Exit Sample has 304,954 patient-year observations.

*** $p<0.01,{ }^{* *} p<0.05,{ }^{*} p<0.1$ 
Table 4: Organizational concentration and quality-related utilization

\begin{tabular}{lcc}
\hline \hline & $(1)$ & $(2)$ \\
& $\begin{array}{c}\text { Mean of } \\
\text { Cependent variable }\end{array}$ & OrgConc $c_{i t}$ \\
\hline $\begin{array}{l}\text { Dependent variable: } \\
\text { A. Hospital outcomes } \\
\text { Any inpatient visit }\end{array}$ & 0.145 & \\
& & -0.085 \\
Any emergency department visit & 0.252 & $-0.137)$ \\
& & $(0.151)$ \\
B. Diabetes care outcomes & & $1.078^{* * *}$ \\
Any HbA1C test & 0.631 & $(0.264)$ \\
& & $1.111^{* * *}$ \\
Any LDL test & 0.590 & $(0.276)$ \\
& & $-0.313^{* * *}$ \\
C. Imaging and testing outcomes & & $(0.072)$ \\
Any outpatient testing or imaging & 0.951 & $-1.236^{* *}$ \\
& & $(0.533)$ \\
\hline
\end{tabular}

Notes: Each row corresponds to a regression. The specifications match that reported in column (4) of Table 3, but with different outcome variables. All regressions control for changes in PCP provider concentration, $\mathrm{PCP}$ characteristics, $\mathrm{PCP}$ organization size, as well as calendar year fixed effects, relative year fixed effects, patient fixed effects. Both changes in PCP organizational concentration and changes in PCP provider concentration are instrumented for using the exiting PCP's practice style. Standard errors are clustered at the PCP and patient levels. Panel A uses the full PCP Exit Sample (304,954 patient-year observations). Panel B uses the subset of the PCP Exit Sample of patients identified with diabetes as chronic condition (106,614 patient-year observations). The first specification in Panel C uses the full PCP Exit Sample (304,954 observations), and the final specification has sample size of 289,979 (only observations with positive utilization of testing and imaging). ${ }^{* * *} p<0.01,{ }^{* *} p<0.05$, * $p<0.1$ 


\section{The Impact of Organizational Boundaries on Healthcare Coordination and Utilization}

Leila Agha, Keith Marzilli Ericson, Xiaoxi Zhao

September 3, 2021

\section{A Online Appendix}

\section{A.1 Sample construction}

Attributing patients to PCPs We attribute each patient to their plurality $\mathrm{PCP}$ in a given year based on the patient's Evaluation \& Management (E\&M) visit in that year. When the number of E\&M visits is tied, we first use the total number of visits; if there is still a tie, we then select randomly.

To index PCP, we adopted the taxonomy classification from Geissler et al. (2020). That classification maps physicians' taxonomy to five groups - primary care, medical specialist, surgical specialist, excluded specialist, non-physician. In this study, we index physicians in the first two groups as PCP.

Identifying PCP exits Our main analysis analyzes two types of PCP exit-relocation and retirement.

To identify PCP relocation events, we first assign each PCP a plurality HRR based on the total number of outpatient visits in a year. About $90 \%$ of PCPs only practice in one HRR each year. We then index PCPs whose plurality HRRs changed as relocating PCPs.

To identify PCP retirements, we looked at the national claims sample. If the last year in which the PCP bills any claims is before the end of our study period-2016, the PCP is identified as retired.

Defining concentration measures Both organizational concentration and provider concentration measures are initially constructed using all outpatient Carrier visits. To identify care delivered in an outpatient setting, we restrict to Carrier claims with place of service listed in Appendix Table A9. About 85\% of visits measured in the Carrier claim file are classified as outpatient care by this definition. 
Defining multispecialty organization By combining claims with the physician specialty taxonomy from Geissler et al. (2020), we develop a definition of a multispecialty organization. For an organization in a year, if at least $10 \%$ of claims are with PCPs and at least $10 \%$ are with non-PCPs, then the organization is indexed as a multispecialty organization.

Utilization decomposition Results reported in Appendix Table A8 decompose total utilization into several components of interest. To investigate the effect of organizational care concentration on different types of utilization, we separate the total utilization into inpatient, outpatient office visits, emergency department, testing and imaging, and other types of outpatient.

- Inpatient utilization: This outcome combines all claims from the Inpatient file, along with Carrier file claims that indicate inpatient hospital place of service (place of service code is "23 Inpatient Hospital").

- Outpatient visits: This outcome combines claims from the Carrier and Outpatient files. Carrier file claims are included here if they do not meet the criteria below for imaging/testing or emergency department care and the care was provided in an office or an hospital outpatient department (places of service code equal to "11 Office" or "22 Outpatient Hospital").

- Testing and imaging: This outcome is from the Carrier and Outpatient files. They are constructed using the Berenson-Eggers Type of Service (BETOS) codes and revenue center codes. More specifically, for Carrier claims, an imaging or lab test is a claim with BETOS code starting with "I" or "T" and place of service is outpatient. For claims in the Outpatient files, they are included if the revenue center codes are in Appendix Table A10.

- Emergency department: This outcome is based on the Carrier and Outpatient files. For Carrier claims, they are included if the place of service is Emergency room at hospital or the procedures codes indicate emergency department (HCPCS codes 99281-99285). Outpatient claims are included if the revenue center indicates emergency room or professional fee related to emergency room. (i.e. revenue center codes 0450-0459 or 0981)

Preventive care measurement The preventive service outcomes reported in Appendix Table A7 were constructed using HCPCS codes and ICD codes, following the procedure defined by Curto et al. (2019). Some of the preventive services we study are recommended by 
the US Preventive Services Task Force (USPTF), with age- and/or gender-specific guidelines. For any guideline with evidence level "C" or better, we limit the sample to the USPTF recommended population. ${ }^{15}$

More specifically

- Mammogram: recommended by USPTF for women age 50-74.

We limit to women ages 65-74.

- Pap smear: not recommended by USPTF for women over 65.

We limit to women (any age).

- Pelvic exam: not recommended by USPTF for women over 65.

We limit to women (any age).

- Prostate cancer screening: recommended by USPTF for men age 55-69.

We limit to men age 65-69.

- Flu vaccine: no USPTF guideline governs flu vaccination, but indicated annually for all ages and genders.

No sample restrictions.

- Colorectal cancer screening: recommended by USPTF for age 45-84.

We limit to $65-84$.

- Cardiovascular screening: no USPTF guideline exists governing cardiovascular screening of this type (including blood cholesterol level tests.)

No sample restrictions.

\section{A.2 Organization Identifiers}

We use Tax Identifcation Numbers (TINs) to identify organizations in our main analysis, but TINs are not a perfect measure of organizational boundaries. Large health systems could use more than one TIN for payments. Using data from 2016, we compare TIN affiliations with another organization identifier — the Group Practice ID (PAC ID) assigned by PECOS and reported in the Physician Compare file.

For most organizations, we could map the two IDs one-to-one. Among physicians affiliated with one organization, $94.5 \%$ of PAC IDs have only one TIN. For the impact of the ID definition on our organizational concentration measure, Appendix Figure A5 shows the

\footnotetext{
${ }^{15}$ USPTF guidelines are available here: https://www.uspreventiveservicestaskforce.org/uspstf/ topic_search_results?topic_status=P. Accessed Aug. 12, 2021.
} 
percentiles of organizational concentration using the two different identifiers. The dots lay very close to the 45-degree line (the green line), showing that there is a very high correlation between the percentile of TIN and PAC IDs.

We were particularly concerned about the possibility of ID-induced measurement error for large physician networks. Physician networks often have multiple TINs, although they contract as a single organization. Based on network comparisons from Geissler et al. (2020), we investigated three large contracting networks in Massachusetts that have the greatest tendency to keep referrals within the network, suggesting a functional organizational identity with practical effects on care patterns. For these three networks, Atrius Health, Fallon Clinic (Reliant Medical Group), and Southcoast Physicians Network, we found 1141, 479, and 433 individual health care providers, respectively. Further, although all three networks have multiple TINs, $98 \%-99 \%$ of claims for affiliated physicians were billed to one TIN for each network. This provides reassuring evidence that TINs provide a useful measure of organizational boundaries that aligns closely with alternative definitions. 
A.3 Appendix Tables and Figures 
Figure A1: Relationship between Organizational Concentration and Healthcare Utilization (residualized by provider concentration).

(A) HRR level

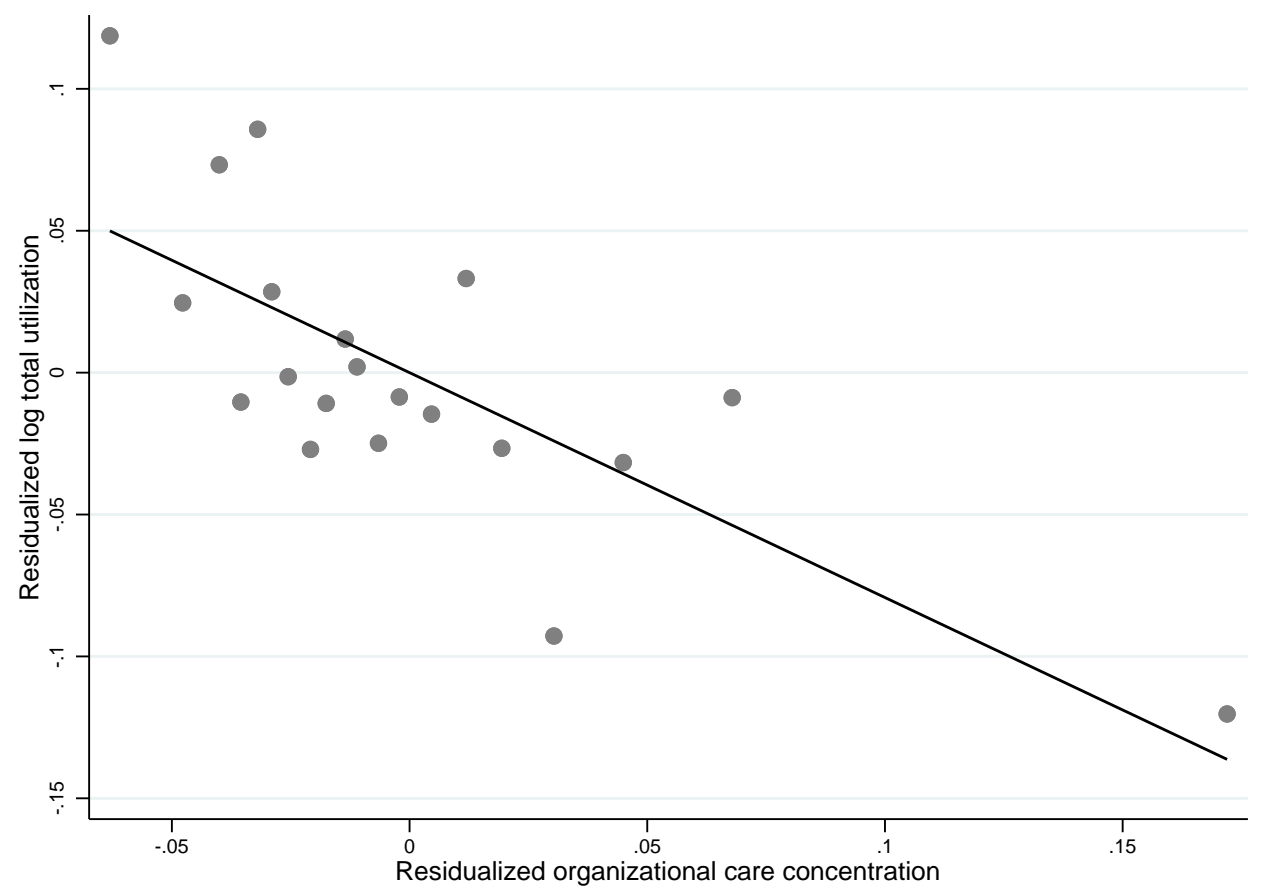

(B) PCP level

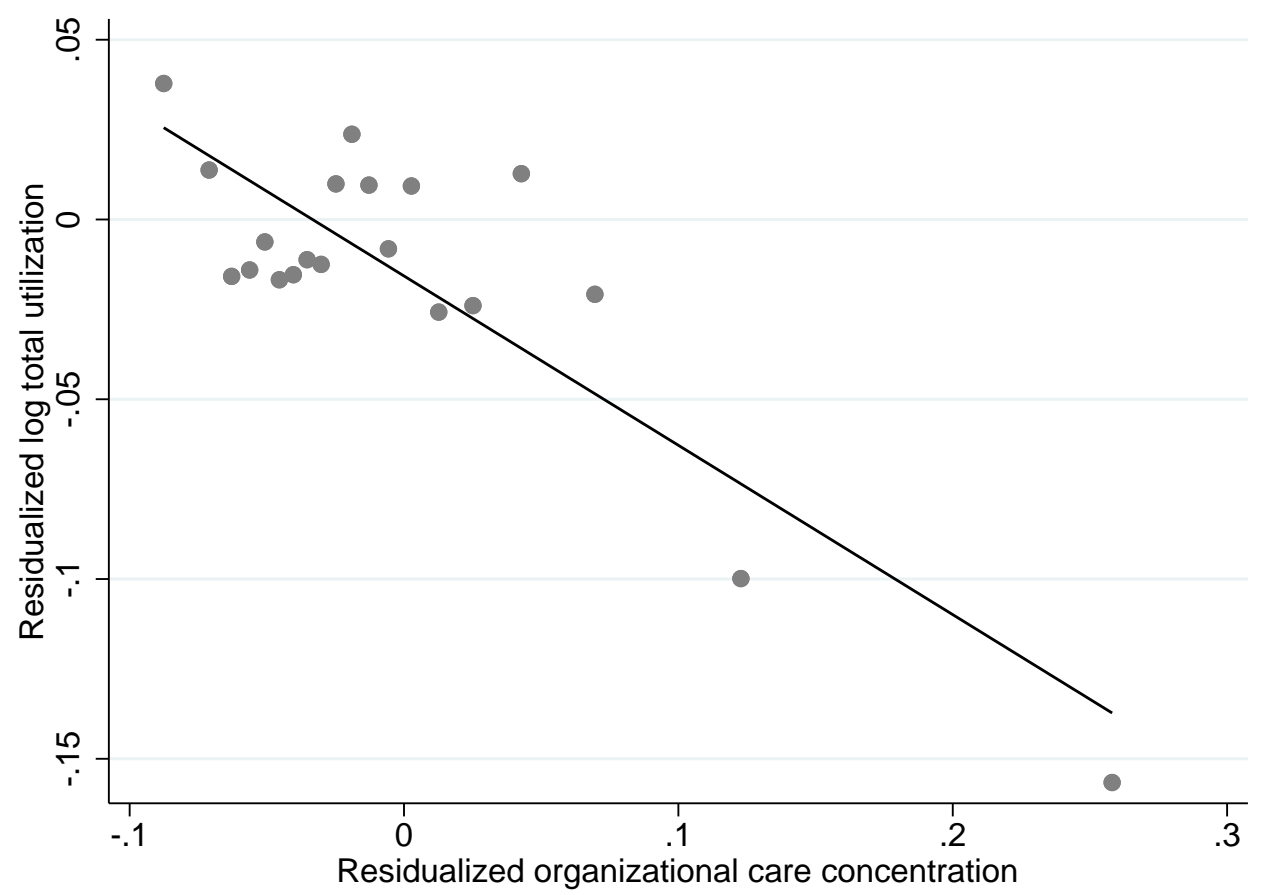

Notes: These binned scatterplots show the relationship between residualized organizational concentration and residualized total healthcare utilization. Panel (A) shows the relationship between these measures averaged at the Hospital Referral Region level. Panel (B) shows the relationship between these measures averaged at the PCP level: an observation is a PCP, an $\oint_{\text {displays the average log utilization and organizational }}$ concentration of their attributed patients. 
Figure A2: Histograms of change in PCP concentration before and after PCP exit

(A) Distribution of change in PCP Organizational Concentration

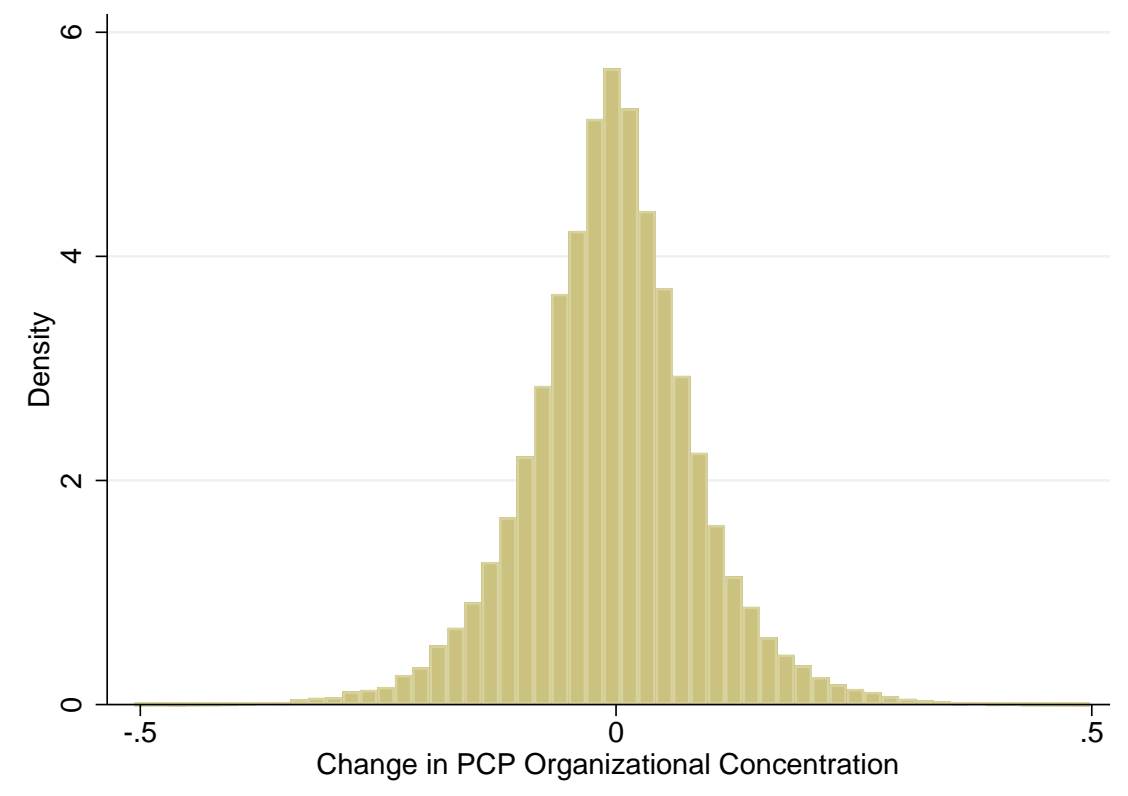

(B) Distribution of change in PCP Provider Concentration

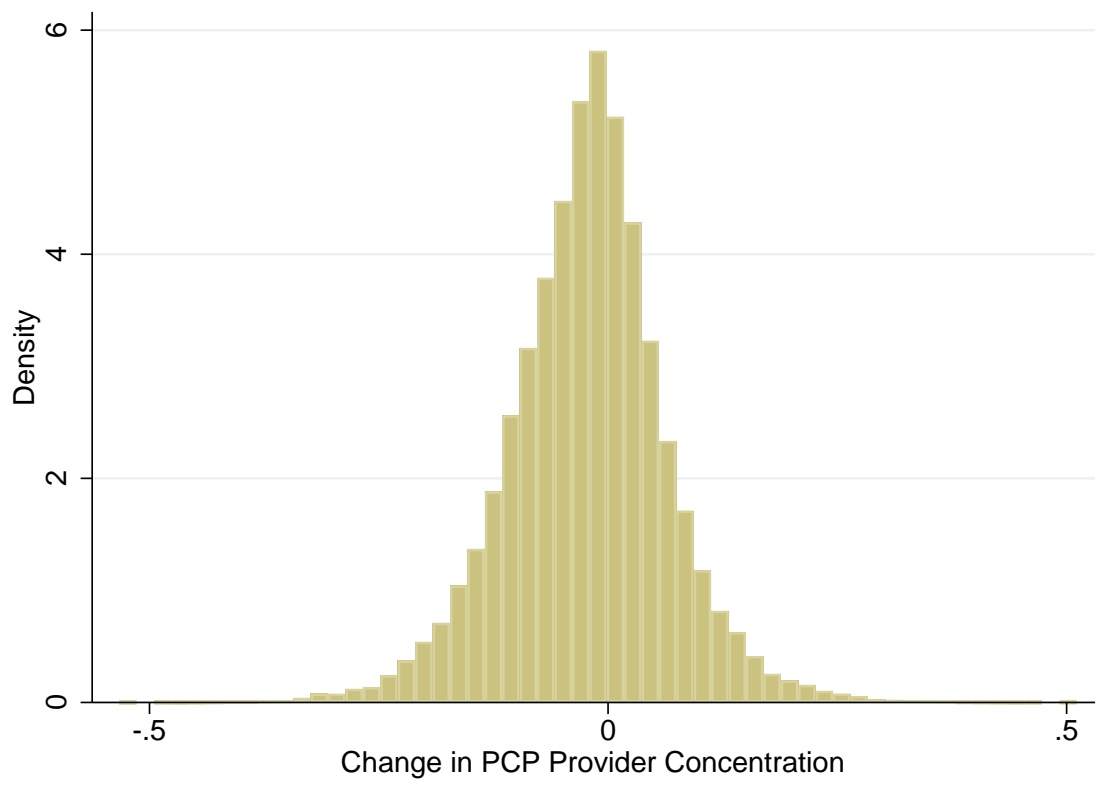

Notes: The two subplots show histograms illustrating the distribution of changes in PCP concentration measures after vs. before PCP exit. These numbers are calculated as the concentration measure of the patient's plurality PCP in the period +1 after their PCP exits minus the concentration measure of their exiting PCP in period -1 . 
Figure A3: Simple binscatter illustrating the identifying variation

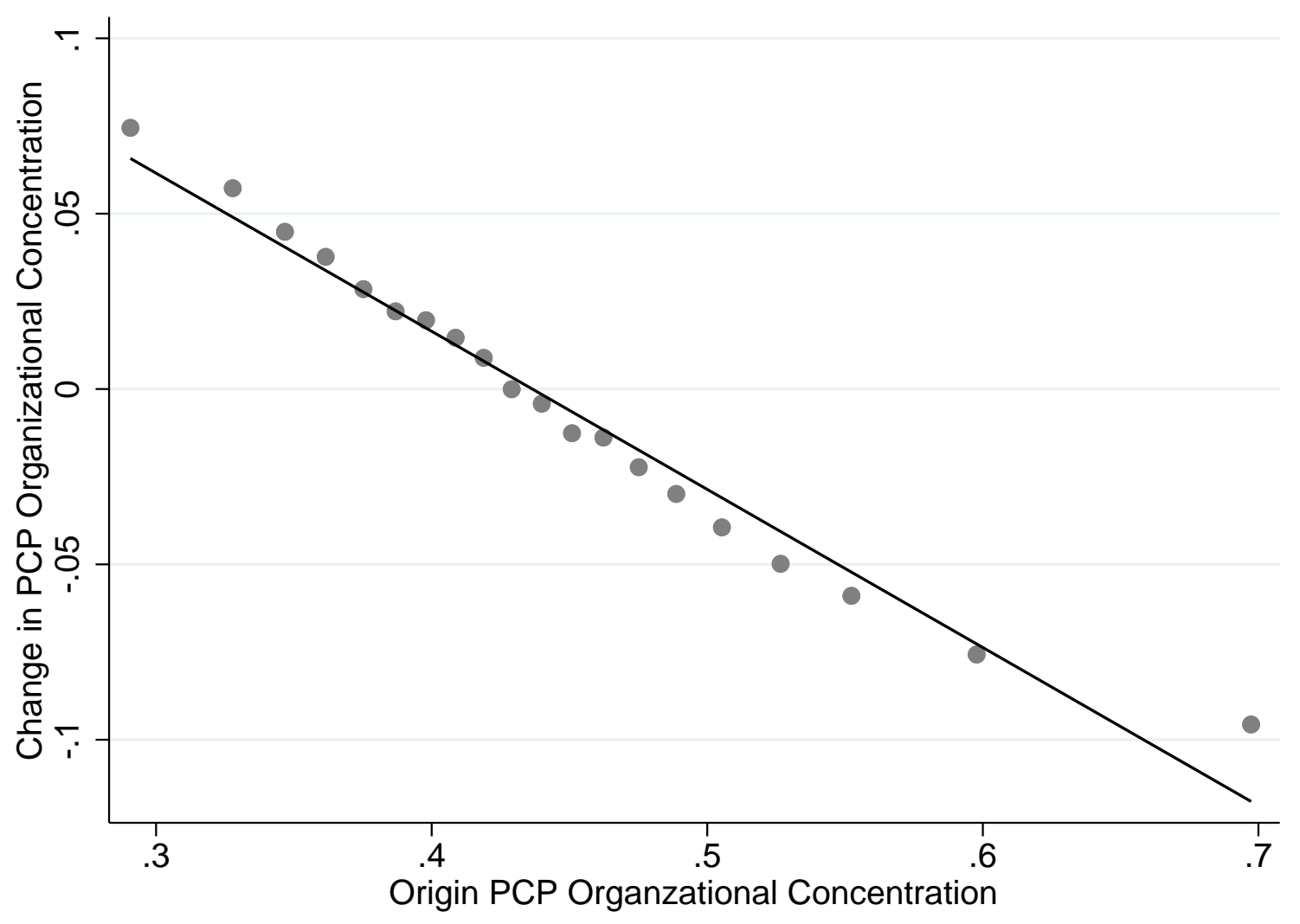

Notes: The figure illustrates the variation underlying the first stage of our IV regressions. It shows that patients whose original PCP has higher organizational concentration on average experience a decrease in their PCP's organizational concentration after the original PCP exits. Panel B illustrates the reduced form, showing that patients whose original PCP had higher organizational concentration on average experience an increase in total utilization after the original PCP exits. 
Figure A4: PCP EXIT EVEnt STUdy, SPLIT By LEVEL OF ORIGIN PCP CONCENTRATION

A. Origin PCP has low organizational concentration
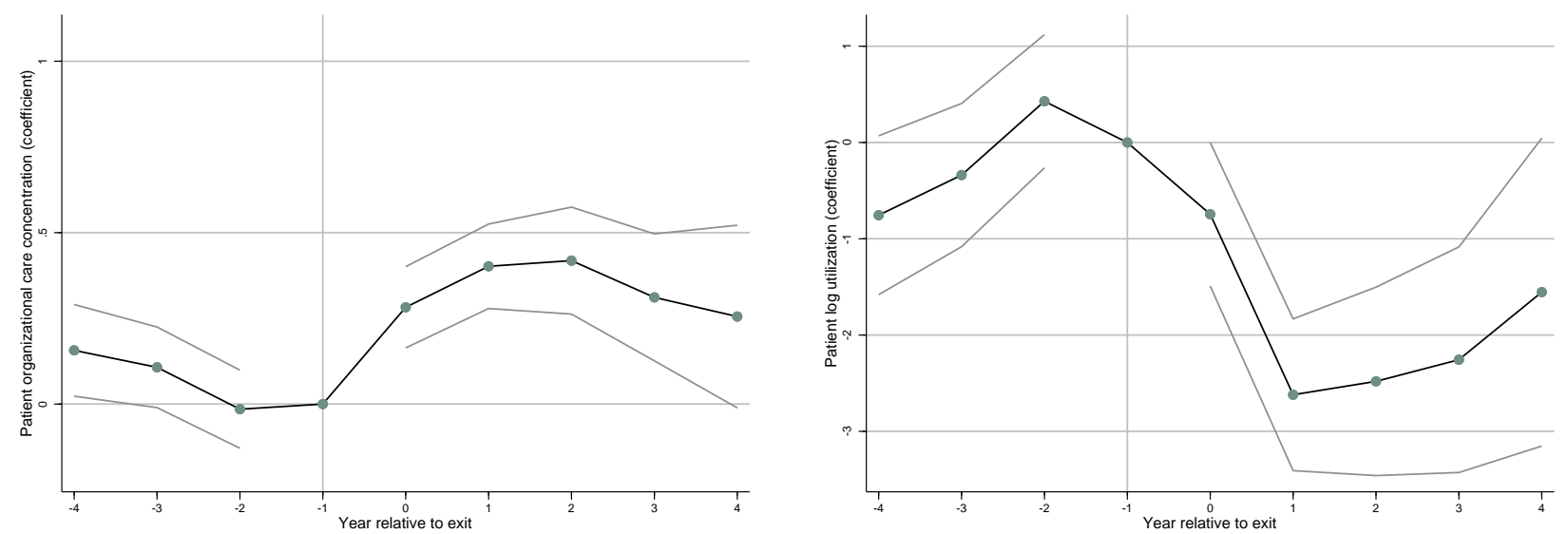

B. Origin PCP has high organizational concentration
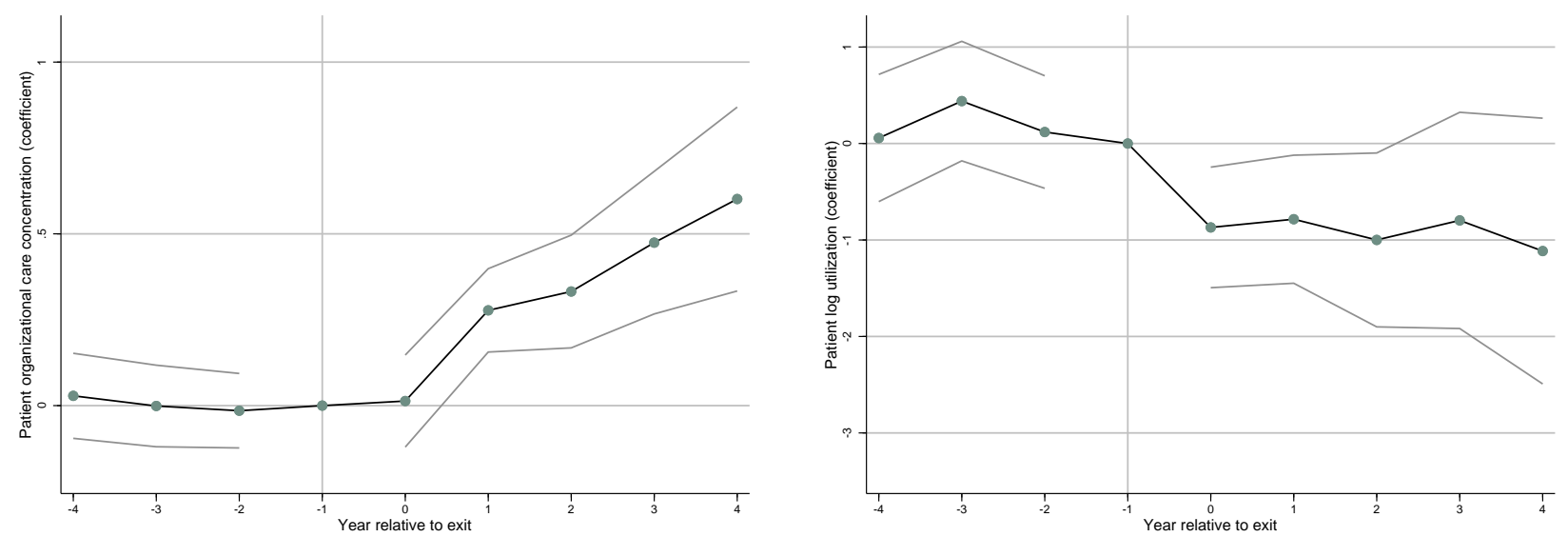

Notes: These plots parallel results shown in Figure 5, but estimated on restricted samples. In Panel A, the sample includes only patients whose origin PCP had below-median organizational concentration; these patients are likely to have experienced an increase in organizational concentration after their original PCP exits. In Panel B, the sample includes only patients whose origin PCP had above-median organizational concentration; these patients are likely to have experienced a decrease in organizational concentration after their original PCP exits. Panel A specifications have 175,390 observations; Panel B specifications have 175,392 observations. 
Figure A5: Q-Q plot of organizational concentration

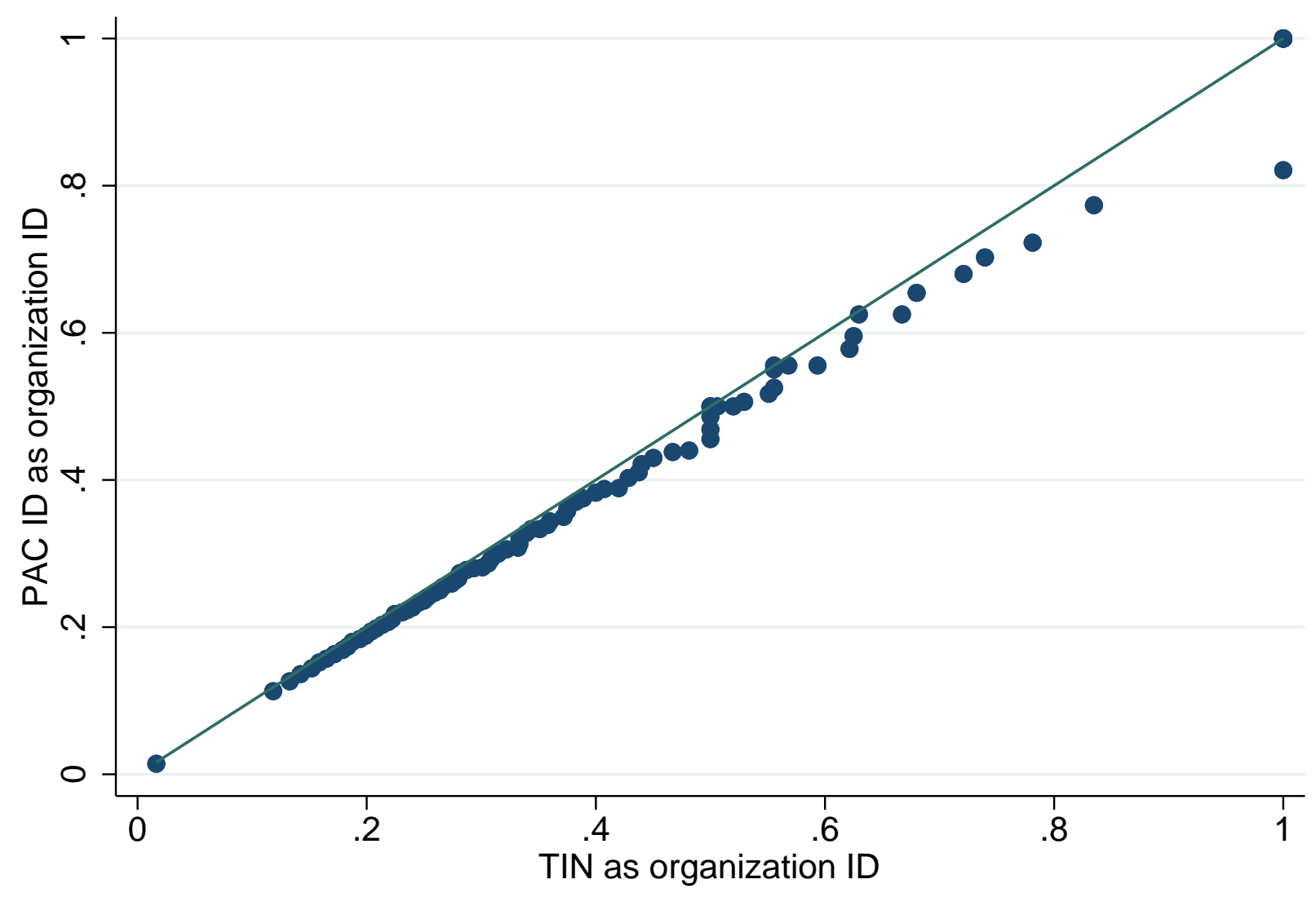

Unit of observation $=$ patient-year

Notes: This figure compares the percentiles of organizational concentration measure using PAC ID as organization ID to the percentiles using TIN as organization ID. The line is the 45-degree line. Each dot is a percentile. 
Table A1: Standard deviation of concentration measures

\begin{tabular}{lc}
\hline \hline & Std. Dev. \\
\hline A. Patient level & \\
$\quad$ Organizational concentration & 0.244 \\
$\quad$ Provider concentration & 0.248 \\
B. PCP level & \\
Organizational concentration (after E.B. shrinkage) & 0.096 \\
Organizational concentration (raw) & 0.108 \\
Provider concentration (after E.B. shrinkage) & 0.083 \\
Provider concentration (raw) & 0.095 \\
C. Hospital referral regional level & \\
Organizational concentration & 0.047 \\
Provider concentration & 0.028 \\
\hline \hline
\end{tabular}

Notes: This table summarizes the variation in provider concentration and organization concentration at different levels of aggregation. Panel A reports the standard deviation of patient-level concentration measures ( $\mathrm{N}=9,177,819)$. Panel B reports the standard deviation of PCP-level concentration measures before and after Empirical Bayes shrinkage (based on 2012 concentration levels for 615,148 PCPs). Panel C reports the standard deviation of region-level concentration measures. In all three panels, there is one observation per patient, so high volume PCPs (in Panel B) and regions (in Panel C) have greater weight in this calculation. 
Table A2: Additional specifications of PCP exit analysis

\begin{tabular}{|c|c|c|c|c|}
\hline \multirow{6}{*}{$\operatorname{OrgConc}_{i, \text { orig }}^{P C P} \times$ post $_{i t}$} & \multicolumn{4}{|c|}{ A. Reduced form results } \\
\hline & \multirow{2}{*}{$\begin{array}{c}(1) \\
\text { OrgConc } \\
\text { Ort }\end{array}$} & \multirow{2}{*}{\multicolumn{3}{|c|}{$\begin{array}{l}(3) \\
\log (\text { total utilization })_{i t}\end{array}$}} \\
\hline & & & & \\
\hline & $\begin{array}{c}-0.204^{* * *} \\
(0.010)\end{array}$ & $\begin{array}{c}0.733^{* * *} \\
(0.053)\end{array}$ & $\begin{array}{c}0.412^{* * *} \\
(0.065)\end{array}$ & $\begin{array}{c}0.459^{* * *} \\
(0.066)\end{array}$ \\
\hline & \multicolumn{4}{|c|}{ B. Difference in differences results } \\
\hline & $(5)$ & $(6)$ & $(7)$ & $(8)$ \\
\hline \multirow[b]{2}{*}{$\Delta \operatorname{OrgConc}_{P C P(i)} \times$ post $_{i t}$} & OrgConc $c_{i t}$ & \multicolumn{3}{|c|}{$\log (\text { total utilization })_{i t}$} \\
\hline & $\begin{array}{c}0.402^{* * *} \\
(0.010)\end{array}$ & $\begin{array}{c}-1.023^{* * *} \\
(0.058)\end{array}$ & $\begin{array}{c}-0.228^{* * *} \\
(0.083)\end{array}$ & $\begin{array}{r}-0.307^{* *} \\
(0.087)\end{array}$ \\
\hline PCP provider concentration & & & $\mathrm{X}$ & $\mathrm{X}$ \\
\hline PCP characteristics \& org. size & & & & $\mathrm{X}$ \\
\hline
\end{tabular}

Notes: In Panel A, this table shows the results of the reduced form regressions underlying the instrumental variable results reported in Table 3. In Panel B, this table estimates a difference in differences equation without using the instrumental variable strategy to predict variation in the change in organizational concentration after a PCP exit. For the difference in differences specification, the key independent variable of interest is the change in the patient's PCPs' organizational concentration one year after the exit minus one year before the exit. All specifications (in both panels) control for calendar year fixed effects, event time fixed effects, and patient fixed effects. In specifications 1 and 5 , the outcome variable is the individual patient's realized organizational concentration and in specifications 2-4 and 6-8 the outcome variable is the patient's log of total utilization. Specifications 3 and 4 include a second instrumental variable: original PCP's provider concentration multiplied by a post indicator. Specifications 7 and 8 also control for the change in PCP provider concentration. Specifications 4 and 8 controls for PCP characteristics: gender, experience quintile indicators, training indicators (internal medicine vs. family practice), and the PCP's organization size (log total number of claims billed to the PCP's TIN, and the log number of unique providers billing to the PCP's TIN). Standard errors have two-way clustering at PCP and patient levels.

*** $p<0.01,{ }^{* *} p<0.05,{ }^{*} p<0.1$ 
Table A3: Impact of organizational concentration and provider concentration

\begin{tabular}{lcccc}
\hline \hline Instrumental Variables & & & & \\
Second stage & $(1)$ & $(2)$ & $(3)$ & $(4)$ \\
& ProvConc $_{i t}$ & $\log (\text { total utilization })_{i t}$ \\
\cline { 2 - 5 } OrgConc $_{i t}^{P C P}$ & & & $-1.625^{* * *}$ & $-2.385^{* * *}$ \\
& & & $(0.287)$ & $(0.440)$ \\
ProvConc $_{i t}^{P C P}$ & $0.787^{* * *}$ & $-1.880^{* * *}$ & $-0.652^{* *}$ & 0.303 \\
& $(0.023)$ & $(0.131)$ & $(0.263)$ & $(0.416)$ \\
First stage F-stat. & & & & \\
& 57,293 & 57,293 & 8951 & 4648 \\
PCP characteristics \& org. size & & & & $\mathrm{X}$ \\
\hline \hline
\end{tabular}

Notes: This table reports the results of instrumental variables regressions similar to those reported in Table 3 , but now providing further detail on the relationship between PCP provider concentration and care utilization. Column 1 reports a specification similar to that in column 1 of Table 3, but replacing the endogenous and instrumental variables related to PCP organizational concentration with analogous variables describing PCP provider concentration, and changing the outcome variable to be patient-level provider concentration. Columns 3 and 4 are identical to the specifications reported in columns 3 and 4 of Table 3 , which include both PCP organizational concentration and PCP provider concentration as endogenous variables, but here we report the coefficient on PCP provider concentration. There are 304,954 patient-year observations. Standard errors have two-way clustering at PCP and patient levels. See notes to Table 3 for further details.

$* * * p<0.01,{ }^{* *} p<0.05,{ }^{*} p<0.1$ 
Table A4: Instrumental variable analysis of PCP exits, additional controls

\begin{tabular}{|c|c|c|c|}
\hline \multicolumn{4}{|l|}{ Instrumental Variables } \\
\hline \multirow[t]{2}{*}{ Second stage } & (1) & (2) & (3) \\
\hline & \multicolumn{3}{|c|}{$\log (\text { total utilization })_{i t}$} \\
\hline $\operatorname{OrgConc} c_{i t}^{P C P}$ & $\begin{array}{c}-2.385^{* * *} \\
(0.440)\end{array}$ & $\begin{array}{c}-2.255^{* * *} \\
(0.418)\end{array}$ & $\begin{array}{c}-2.215^{* * *} \\
(0.427)\end{array}$ \\
\hline First stage F-stat. & 4648 & 5073 & 4882 \\
\hline PCP provider conc. & $\mathrm{X}$ & $\mathrm{X}$ & $\mathrm{X}$ \\
\hline PCP characteristics & $\mathrm{X}$ & $\mathrm{X}$ & $\mathrm{X}$ \\
\hline PCP org. size (log) & $\mathrm{X}$ & & \\
\hline PCP org. size (5 bins) & & $\mathrm{X}$ & $\mathrm{X}$ \\
\hline PCP multi-specialty practice & & & $\mathrm{X}$ \\
\hline
\end{tabular}

Notes: See notes to Table 3. For reference, specification (1) replicates the results reported in (4) of Table 3. Column 2 substitutes the control for number of physicians and number of claims in the organization with 5 quintile indicator variables for each measure of organization size. Column 3 adds a control variable for multi-specialty practice.

*** $p<0.01,{ }^{* *} p<0.05,{ }^{*} p<0.1$ 
Table A5: Instrumental variable analysis of PCP exits, alternative functional forms for provider concentration

\begin{tabular}{lccc}
\hline \hline Instrumental Variables & & & \\
Second stage & $(1)$ & $(2)$ & $(3)$ \\
& \multicolumn{2}{c}{$\log (\text { total utilization })_{i t}$} \\
\cline { 2 - 4 } OrgConc $_{i t}^{P C P}$ & $-2.385^{* * *}$ & $-2.366^{* * *}$ & $-2.929^{* * *}$ \\
& $(0.440)$ & $(0.439)$ & $(0.363)$ \\
& & & \\
First stage F-stat. & 4648 & 3151 & 4606 \\
& & & \\
PCP provider concentration & $\mathrm{X}$ & $\mathrm{X}$ & $\mathrm{X}$ \\
PCP characteristics & $\mathrm{X}$ & $\mathrm{X}$ & $\mathrm{X}$ \\
PCP organizational size & $\mathrm{X}$ & $\mathrm{X}$ & $\mathrm{X}$ \\
PCP provider concentration quadratic & & $\mathrm{X}$ & \\
Spline N generalists seen by patient & & & $\mathrm{X}$ \\
Spline N specialists seen by patient & & & $\mathrm{X}$ \\
\hline \hline
\end{tabular}

Notes: See notes to Table 3. For reference, specification (1) replicates the results reported in (4) of Table 3. In specification (2), we add a quadratic term in PCP provider concentration as an additional endogenous variable. To identify the model, we add an additional quadratic instrumental variable as well: $\left(\text { OrgConc }_{i, \text { orig }}^{P C P}\right)^{2}$ post $_{i t}$. In specification (3), the regression adds new control variables that account for the number of distinct providers each patient sees. Specifically, these specifications control for a 4-knot spline in the number of generalist providers (as defined in Table A1: family practice, internal medicine training, or geriatrics training) and a 4-knot spline in the number of specialist providers (with any other training type). Standard errors have two-way clustering at PCP and patient levels.

*** $p<0.01,{ }^{* *} p<0.05,{ }^{*} p<0.1$ 
Table A6: Organizational concentration and repeated imaging

\begin{tabular}{ccc}
\hline \hline & $(1)$ & $(2)$ \\
& $\begin{array}{c}\text { Mean of } \\
\text { dependent variable }\end{array}$ & $\begin{array}{c}\text { Coefficient on } \\
\text { OrgConc }_{i t}\end{array}$ \\
\hline Dependent variable: & & \\
Total imaging scans & 1.448 & -1.504 \\
& & $(0.987)$ \\
Total repeated imaging & 0.280 & -0.226 \\
& & $(0.517)$ \\
\hline \hline
\end{tabular}

Notes: Each row corresponds to a regression. The specifications match that reported in column (4) of Table 3, but with alternative dependent variables. Sample size is 304,954 .

*** $p<0.01,{ }^{* *} p<0.05,{ }^{*} p<0.1$ 
Table A7: Organizational concentration and preventive care

\begin{tabular}{|c|c|c|c|}
\hline & $\begin{array}{l}\text { Sample } \\
\text { size }\end{array}$ & $\begin{array}{c}\text { Mean of } \\
\text { dependent variable }\end{array}$ & $\begin{array}{l}\text { Coefficient on } \\
\text { OrgConc } c_{i t}^{P C P}\end{array}$ \\
\hline \multicolumn{4}{|l|}{ Dependent variable: } \\
\hline Mammogram $\dagger$ & 59,022 & 0.695 & $\begin{array}{c}0.352 \\
(0.615)\end{array}$ \\
\hline Pap smear & 189,368 & 0.144 & $\begin{array}{l}-0.218 \\
(0.189)\end{array}$ \\
\hline Pelvic exam & 189,368 & 0.125 & $\begin{array}{c}0.085 \\
(0.168)\end{array}$ \\
\hline Prostate cancer screening $\dagger$ & 12,605 & 0.354 & $\begin{array}{c}0.655 \\
(1.306)\end{array}$ \\
\hline Flu shot & 304,954 & 0.672 & $\begin{array}{c}0.261 \\
(0.216)\end{array}$ \\
\hline Colorectal screening $\dagger$ & 242,103 & 0.163 & $\begin{array}{c}-0.799^{* * *} \\
(0.183)\end{array}$ \\
\hline Cardiovascular screening & 304,954 & 0.905 & $\begin{array}{c}0.297 \\
(0.389)\end{array}$ \\
\hline
\end{tabular}

Notes: Each row corresponds to a regression. The specifications match that reported in column (4) of Table 3 , but with alternative dependent variables.

$\dagger$ indicates a type of care that is recommended by the US Preventive Services Task Force for the age and sex group in the regression sample. For more details on the construction of these outcomes see Appendix A.1.

${ }^{* * *} p<0.01,{ }^{* *} p<0.05,{ }^{*} p<0.1$ 


\section{Table A8: Instrumental variable analysis of PCP exits, spending decomposition}

\begin{tabular}{|c|c|c|c|c|c|c|}
\hline & \multirow[b]{3}{*}{$\begin{array}{c}\text { Mean utilization } \\
\text { (in } \$)\end{array}$} & \multirow[b]{3}{*}{$\begin{array}{c}\text { Sample with }>0 \\
\text { spending (for col. } 3 \text { ) }\end{array}$} & \multicolumn{4}{|c|}{ Dependent variable: } \\
\hline & & & (1) & $(2)$ & $(3)$ & $(4)$ \\
\hline & & & $\begin{array}{l}\text { Utilization } \\
\text { (in } \$)\end{array}$ & $\begin{array}{l}\text { Any utilization } \\
\text { (indicator) }\end{array}$ & $\begin{array}{c}\log (\text { Utilization }) \\
\quad(\text { if }>0)\end{array}$ & $\log (1+$ Utilization $)$ \\
\hline \multicolumn{7}{|l|}{ Spending category: } \\
\hline Total utilization & 7517 & 304,953 & $\begin{array}{c}-14,088^{* * *} \\
(5399)\end{array}$ & & $\begin{array}{c}-2.385^{* * *} \\
(0.440)\end{array}$ & $\begin{array}{c}-2.387^{* * *} \\
(0.440)\end{array}$ \\
\hline Inpatient (hosp. \& prof.) & 3008 & 44,245 & $\begin{array}{l}-5867 \\
(3870)\end{array}$ & $\begin{array}{l}-0.085 \\
(0.137)\end{array}$ & $\begin{array}{c}0.097 \\
(1.448)\end{array}$ & $\begin{array}{l}-1.107 \\
(1.248)\end{array}$ \\
\hline Outpatient visits (hosp. \& prof.) & 2346 & 304,854 & $\begin{array}{l}-438 \\
(1924)\end{array}$ & $\begin{array}{c}-0.010^{* *} \\
(0.004)\end{array}$ & $\begin{array}{c}-1.707^{* * *} \\
(0.357)\end{array}$ & $\begin{array}{c}-1.767^{* * *} \\
(0.358)\end{array}$ \\
\hline Outpatient testing \& imaging (hosp. \& prof.) & 1351 & 289,979 & $\begin{array}{c}-4202^{* *} \\
(1759)\end{array}$ & $\begin{array}{c}-0.313^{* * *} \\
(0.072)\end{array}$ & $\begin{array}{c}-1.236^{* *} \\
(0.533)\end{array}$ & $\begin{array}{c}-2.675^{* * *} \\
(0.635)\end{array}$ \\
\hline Emergency department & 357 & 76,854 & $\begin{array}{c}-1009^{* *} \\
(407)\end{array}$ & $\begin{array}{l}-0.273^{*} \\
(0.151)\end{array}$ & $\begin{array}{c}-1.787^{* * *} \\
(0.641)\end{array}$ & $\begin{array}{c}-2.365^{* *} \\
(1.006)\end{array}$ \\
\hline Other (incl. home health, urgent care, etc.) & 454 & 226,637 & $\begin{array}{l}-516 \\
(381)\end{array}$ & $\begin{array}{c}-1.581^{* * *} \\
(0.174)\end{array}$ & $\begin{array}{c}-2.825^{* * *} \\
(0.623)\end{array}$ & $\begin{array}{c}-9.579^{* * *} \\
(1.067) \\
\end{array}$ \\
\hline
\end{tabular}

Notes: See notes to Table 3. This table replicates the instrumental variable specification reported in Table 3 specification (4) with alternative outcome variables that decompose Medicare billing depending on the type of bill. For details on how we define each category of spending, see Appendix A.1. The full sample size is 304,954 ; column 3 sample size varies by row and is reported in the table.

$* * * p<0.01, * * p<0.05, * p<0.1$ 
Table A9: List of place of service codes included as outpatient care

\begin{tabular}{cl}
\hline \hline Place of Service Code & \multicolumn{1}{c}{ Place of Service Name } \\
\hline 05 & Indian Health Service Free-standing Facility \\
07 & Tribal 638 Free-standing Facility \\
11 & Office \\
17 & Walk-in Retail Health Clinic \\
20 & Urgent Care Facility \\
22 & On Campus-Outpatient Hospital \\
49 & Independent Clinic \\
50 & Federally Qualified Health Center \\
53 & Community Mental Health Center \\
57 & Non-residential Substance Abuse Treatment Facility \\
58 & Non-residential Opioid Treatment Facility \\
62 & Comprehensive Outpatient Rehabilitation Facility \\
65 & End-Stage Renal Disease Treatment Facility \\
71 & Public Health Clinic \\
72 & Rural Health Clinic \\
\hline \hline
\end{tabular}

Notes: These codes are used to identify claims in the Medicare Carrier File for services that take place in an outpatient facility. 
Table A10: List of revenue center codes included as testing and imaging codes

\begin{tabular}{ll}
\hline \hline Revenue Center Code & Short description \\
\hline $0300-0319$ & Laboratory \\
$0320-0329$ & Radiology diagnostic \\
$0400-0409$ & Other imaging services \\
0482 & Cardiology-stress test \\
0483 & Cardiology-Echocardiology \\
$0610-0619$ & Magnetic resonance technology \\
$0730-0749$ & EKG/ECG \\
0971 & Professional fees-laboratory \\
0972 & Professional fees-radiology diagnostic \\
\hline \hline
\end{tabular}

Notes: These revenue center codes are used to identify outpatient testing and imaging claims. 